KCL-MTH-08-08

ZMP-HH/08-17

Hamburger Beiträge zur Mathematik Nr. 320

Dezember 2008

\title{
MODULE CATEGORIES FOR PERMUTATION MODULAR INVARIANTS
}

\author{
Till Barmeier ${ }^{a}$, Jürgen Fuchs ${ }^{b}$, Ingo Runkel ${ }^{c}$, Christoph Schweigert ${ }^{a \circledast}$ \\ ${ }^{a}$ Organisationseinheit Mathematik, Universität Hamburg \\ Schwerpunkt Algebra und Zahlentheorie \\ Bundesstraße 55, D-20146 Hamburg \\ b Teoretisk fysik, Karlstads Universitet \\ Universitetsgatan 21, S-65188 Karlstad \\ c Department of Mathematics, King's College London \\ Strand, UK-London WC2R 2LS
}

\begin{abstract}
We show that a braided monoidal category $\mathcal{C}$ can be endowed with the structure of a right (and left) module category over $\mathcal{C} \times \mathcal{C}$. In fact, there is a family of such module category structures, and they are mutually isomorphic if and only if $\mathcal{C}$ allows for a twist. For the case that $\mathcal{C}$ is premodular we compute the internal End of the tensor unit of $\mathcal{C}$, and we show that it is an Azumaya algebra if $\mathcal{C}$ is modular. As an application to two-dimensional rational conformal field theory, we show that the module categories describe the permutation modular invariant for models based on the product of two identical chiral algebras. It follows in particular that all permutation modular invariants are physical.
\end{abstract}

\footnotetext{
* Email addresses:

barmeier@math.uni-hamburg.de, jfuchs@fuchs.tekn.kau.se, ingo.runkel@kcl.ac.uk, schweigert@math.uni-hamburg.de
} 


\section{Introduction}

Just as a monoidal category $\mathcal{X}$ can be understood as the categorification of a ring, a right module category $\mathcal{M}$ is the categorification of a right module over a ring. Namely, there is a bifunctor $\bigotimes: \mathcal{M} \times \mathcal{X} \rightarrow \mathcal{M}$ together with associativity and unit constraints obeying suitable compatibility conditions (they will be recalled in section 2 below). Module categories are for instance used to define Morita equivalence between monoidal categories [Mü], and they play a significant role in the study of two-dimensional rational conformal field theory [SFR].

Any ring can be regarded as a right module over itself by setting $m \cdot a:=m \cdot a$. In the same way, the tensor product and the associativity constraints can be used to endow any monoidal category $\mathcal{C}$ with the structure of a right module category over itself. A commutative ring $R$ carries the structure of a right module over the ring $R \otimes_{\mathbb{Z}} R$ by $a .(b \otimes c):=a \cdot b \cdot c$. Notice that the module property requires the equality

$$
\begin{aligned}
m \cdot a_{1} \cdot a_{2} \cdot b_{1} \cdot b_{2} & \equiv m \cdot\left(\left(a_{1} \otimes b_{1}\right) \cdot\left(a_{2} \otimes b_{2}\right)\right) \\
& =\left(m \cdot\left(a_{1} \otimes b_{1}\right)\right) \cdot\left(a_{2} \otimes b_{2}\right) \equiv m \cdot a_{1} \cdot b_{1} \cdot a_{2} \cdot b_{2},
\end{aligned}
$$

which holds by commutativity of $R$. The categorification of a commutative ring is an additive braided monoidal category. Hence the question arises whether any braided monoidal category $\mathcal{C}$ carries the structure of a right module category over the monoidal category $\mathcal{C} \times \mathcal{C}$. We show that this is indeed the case. However, the lift of the permutation to an element in the braid group is not unique. Using inequivalent lifts, we present a family of module category structures, indexed by integers, on these categories and show that they are mutually equivalent if and only if $\mathcal{C}$ can be equipped with a twist, or balancing, see Theorems 2 and 1 and Remark 7 .

Given a module category $\mathcal{M}$ over $\mathcal{X}$, denote by $\mathcal{E} n d_{\mathcal{X}}(\mathcal{M})$ the category of module endofunctors of $\mathcal{M}$. This is a monoidal category, with composition as tensor product. We recall in section 3 that if $\mathcal{X}$ is braided there are two monoidal functors $\alpha^{ \pm}: \mathcal{X} \rightarrow \mathcal{E} n d_{\mathcal{X}}(\mathcal{M})$ called braided induction. In section 7 we show that if $\mathcal{C}$ is a modular tensor category, and if we take the module category to be $\mathcal{M}=\mathcal{C}$ over $\mathcal{X}=\mathcal{C} \otimes \mathcal{C}$, i.e. the completion of $\mathcal{C} \times \mathcal{C}$ with respect to direct sums, then $\alpha^{ \pm}$are monoidal equivalences.

The proof proceeds in two steps. We first compute End(1), the internal End of the monoidal unit $\mathbf{1} \in \mathcal{O} b j(\mathcal{C})$. Assuming that $\mathcal{C}$ is premodular (see Section $\mathbb{Q}$ ), the internal End of any object of $\mathcal{M}$ exists and is an algebra in $\mathcal{C} \otimes \mathcal{C}$, and the category of modules over this algebra is equivalent, as a module category over $\mathcal{C} \nabla \mathcal{C}$, to $\mathcal{C}$. We also show, in Section 6, that the algebra End(1) has the structure of a (symmetric special) Frobenius algebra. If $\mathcal{C}$ is even a modular tensor category, i.e. if it is premodular and the braiding obeys a certain non-degeneracy condition, we show that End(1) is Azumaya. An algebra in a braided monoidal category is an Azumaya algebra iff the braided induction functors $\alpha^{ \pm}$are monoidal equivalences, see section 7. In Lemma 10 we establish an alternative criterion for an algebra to be Azumaya, and in Proposition 16 we verify that this criterion is met for End(1). We also show that the composition $\left(\alpha^{-}\right)^{-1} \circ \alpha^{+}$is naturally equivalent to the functor that permutes the two factors in $\mathcal{C} \times \mathcal{C}$ (Lemma 8 and Proposition 18).

The latter result has an application to conformal field theory. Let $\mathcal{V}$ be a vertex operator algebra satisfying the conditions of Theorem 2.1 of [Hu], according to which the category $\mathcal{C}$ of representations of $\mathcal{V}$ is a modular tensor category. Now the product $\mathcal{V} \otimes_{\mathbb{C}} \mathcal{V}$ satisfies the conditions of that theorem as well, and its representations form the category $\mathcal{C} \otimes \mathcal{C}$. Further, by [FRS1, FjFRS1, a full conformal field theory with left and right chiral symmetry given by the product 
$\mathcal{V} \otimes_{\mathbb{C}} \mathcal{V}$ is described by a (symmetric special) Frobenius algebra in $\mathcal{C} \otimes \mathcal{C}$. If this Frobenius algebra is Azumaya, then the modular invariant torus partition function of the full conformal field theory is of permutation type. Our results imply that the algebra End $(\mathbf{1})$ realises the conformal field theory associated to the transposition symmetry of the two factors in $\mathcal{V} \otimes_{\mathbb{C}} \mathcal{V}$. This is non-trivial because a modular invariant bilinear combination of characters is not automatically the partition function of a physical conformal field theory with a consistent collection of correlators. As an immediate corollary, we can also obtain algebras that implement the modular invariants for $\mathcal{C}^{\otimes N}$ corresponding to arbitrary transpositions in $\mathfrak{S}_{N}$. Since any permutation in $\mathfrak{S}_{N}$ can be written as a product of transpositions, and since the tensor product of algebras implements, by Proposition 5.3 of [FRS1, the product of modular invariants, our results imply that all permutation modular invariants in rational conformal field theory are physical.

From this point of view another question is natural: What is the chiral conformal field theory whose chiral symmetry consists of the fixed algebra $\left(\mathcal{V} \otimes_{\mathbb{C}} \mathcal{V}\right)^{\mathfrak{S}_{2}}$ under the action of the permutation group $\mathfrak{S}_{2}$ on $\mathcal{V} \otimes_{\mathbb{C}} \mathcal{V}$ ? Theories for which the chiral symmetry is given by a fixed algebra are called orbifold models. On the category theoretical side, the orbifold by a symmetry group $G$ can be obtained by starting from a $G$-equivariant fusion category [Tu , from which one then calculates the orbifold category $\mathcal{X} / G$ [Ki, Mü2]. Part of the data of a $G$-equivariant fusion category consists of a list of categories $\mathcal{X}_{g}$, indexed by elements $g \in G$, together with bifunctors $\mathcal{X}_{g} \times \mathcal{X}_{h} \rightarrow \mathcal{X}_{g h}$ subject to certain compatibility conditions. From the analogy with conformal field theory one expects that an $N$-fold product $\mathcal{C}^{\otimes N}$ can be embedded into an $\mathfrak{S}_{N^{-}}$-equivariant category $\mathcal{X}$ as its neutral component, $\mathcal{X}_{e}=\mathcal{C}^{\otimes N}$. (This can possibly be done in different ways, which are indexed by elements of $H^{2}\left(\mathfrak{S}_{N}, U(1)\right)$.) Our construction provides some of the necessary ingredients for obtaining an $\mathfrak{S}_{2}$-equivariant fusion category with $\mathcal{X}_{e}=\mathcal{C} \otimes \mathcal{C}$ and $\mathcal{X}_{\pi}=\mathcal{C}$, namely, along with $\mathcal{X}_{e} \times \mathcal{X}_{e} \rightarrow \mathcal{X}_{e}$, also the bifunctors $\mathcal{X}_{\pi} \times \mathcal{X}_{e} \rightarrow \mathcal{X}_{\pi}$ and $\mathcal{X}_{e} \times \mathcal{X}_{\pi} \rightarrow \mathcal{X}_{\pi}$ with mixed associators that obey all mixed pentagon relations.

This paper is organised as follows. In section 2 we obtain the family of module category structures of $\mathcal{C}$ over $\mathcal{C} \times \mathcal{C}$, and in section 3 we discuss the braided induction associated to these module categories. For passing from $\mathcal{C} \times \mathcal{C}$ to $\mathcal{C} \otimes \mathcal{C}$ we need to verify that the construction is compatible with direct sums; this is done in section 4 . In section 5 we compute the internal End of $\mathbf{1} \in \mathcal{O} b j(\mathcal{C})$, and in section 7 we show that it is an Azumaya algebra. In section 8 we conclude with some remarks and observations regarding our results.

\section{$2 \quad$ A family of module categories}

We start by recalling some basic notions that will be needed.

A (right) module category $(\mathcal{M}, \bigotimes, \psi, r)$ over a monoidal category $\mathcal{X}=(\mathcal{X}, \otimes, \mathbf{1}, a, \lambda, \rho)$ is $[\mathrm{Os}]$ 开 category $\mathcal{M}$ together with a bifunctor $\otimes: \mathcal{M} \times \mathcal{X} \rightarrow \mathcal{M}$ and natural associativity and unit isomorphisms $\psi_{M, X, Y}: M \otimes(X \otimes Y) \rightarrow(M \otimes X) \otimes Y$ and $r_{M}: M \otimes \mathbf{1} \rightarrow M$. They satisfy the pentagon identity

$$
\psi_{M \otimes X, Y, Z} \circ \psi_{M, X, Y \otimes Z}=\left(\psi_{M, X, Y} \otimes i d_{Z}\right) \circ \psi_{M, X \otimes Y, Z} \circ\left(i d_{M} \otimes a_{X, Y, Z}\right)
$$

${ }^{\dagger}$ In $\mathrm{Os}$ it is assumed that the categories $\mathcal{X}$ and $\mathcal{M}$ are abelian and that the tensor product bifunctor is exact. These assumptions will actually be met in the situation studied further below, in which $\mathcal{X}$ is premodular, but they are not needed here. 
for all $M \in \mathcal{O} b j(\mathcal{M})$ and $X, Y, Z \in \mathcal{O} b j(\mathcal{X})$ and the triangle identity

$$
i d_{M} \otimes \lambda_{X}=\left(r_{M} \otimes i d_{X}\right) \circ \psi_{M, \mathbf{1}, X}
$$

for all $M \in \mathcal{O} b j(\mathcal{M})$ and $X \in \mathcal{O} b j(\mathcal{X})$.

If $\left(\mathcal{M}, \bigotimes_{\mathcal{M}}, \psi^{\mathcal{M}}, r^{\mathcal{M}}\right)$ and $\left(\mathcal{N}, \bigotimes_{\mathcal{N}}, \psi^{\mathcal{N}}, r^{\mathcal{N}}\right)$ are module categories over the same monoidal category $\mathcal{X}$, then a (strict) module functor $(G, \gamma)$ from $\mathcal{M}$ to $\mathcal{N}$ is [OS, Def. 2.7] a functor $G: \mathcal{M} \rightarrow \mathcal{N}$ together with a collection of natural isomorphisms

$$
\gamma_{M, X}: \quad G\left(M \otimes_{\mathcal{M}} X\right) \rightarrow G(M) \otimes_{\mathcal{N}} X
$$

for $M \in \mathcal{O} b j(\mathcal{M})$ and $X \in \mathcal{O} b j(\mathcal{X})$ that obey the following associativity and unit constraints: first, for all $M \in \mathcal{O} b j(\mathcal{M})$ and $X, Y \in \mathcal{O} b j(\mathcal{X})$ one has

$$
\left(\gamma_{M, X} \otimes_{\mathcal{N}} i d_{Y}\right) \circ \gamma_{M \otimes_{\mathcal{M} X, Y}} \circ G\left(\psi_{M, X, Y}^{\mathcal{M}}\right)=\psi_{G(M), X, Y}^{\mathcal{N}} \circ \gamma_{M, X \otimes Y}
$$

as morphisms $G\left(M \otimes_{\mathcal{M}}(X \otimes Y)\right) \rightarrow\left(G(M) \bigotimes_{\mathcal{N}} X\right) \bigotimes_{\mathcal{N}} Y$; and second, for all $M \in \mathcal{O} b j(\mathcal{M})$ one has

$$
G\left(r_{M}^{\mathcal{M}}\right)=r_{G(M)}^{\mathcal{N}} \circ \gamma_{M, 1}
$$

as morphisms $G\left(M \mathbf{\nabla}_{\mathcal{M}} \mathbf{1}\right) \rightarrow G(M)$.

Given two module functors $(G, \gamma)$ and $\left(G^{\prime}, \gamma^{\prime}\right)$ from $\mathcal{M}$ to $\mathcal{N}$, a natural transformation $\eta$ : $(G, \gamma)$ $\rightarrow\left(G^{\prime}, \gamma^{\prime}\right)$ of module functors is a natural transformation $\eta: G \rightarrow G^{\prime}$ that obeys

$$
\left(\eta_{M} \otimes_{\mathcal{N}} i d_{X}\right) \circ \gamma_{M, X}=\gamma_{M, X}^{\prime} \circ \eta_{M \otimes_{\mathcal{M} X}}
$$

for all $M \in \mathcal{M}$ and $X \in \mathcal{X}$. Two module categories $\mathcal{M}$ and $\mathcal{N}$ over $\mathcal{X}$ are called equivalent iff there exists a module functor $(F, \varphi): \mathcal{M} \rightarrow \mathcal{N}$ such that $F$ is an equivalence of categories. One can check that this is the same condition as demanding the existence of a module functor $(G, \gamma): \mathcal{N} \rightarrow \mathcal{M}$ such that $(F, \varphi) \circ(G, \gamma)$ and $(G, \gamma) \circ(F, \varphi)$ are naturally isomorphic to $\left(\mathrm{id}_{\mathcal{N}}, i d\right)$ and $\left(\mathrm{id}_{\mathcal{M}}, i d\right)$, respectively.

Invoking coherence, from here on we adhere to the following

Convention 1. Unless noted otherwise, a monoidal category is assumed to be strict, i.e. to have trivial associativity and unit isomorphisms $a_{X, Y, Z}$ and $\lambda_{X}, \rho_{X}$. Accordingly we will write $\mathcal{X}=(\mathcal{X}, \otimes, \mathbf{1})$ in place of $\mathcal{X}=(\mathcal{X}, \otimes, \mathbf{1}, a, \lambda, \rho)$.

For any two categories $\mathcal{C}$ and $\mathcal{D}$ we denote by $\mathcal{C} \times \mathcal{D}$ the category whose objects are ordered pairs $U \times V$ of objects of $\mathcal{C}$ and $\mathcal{D}$ and whose morphism sets are Cartesian products $\operatorname{Hom}_{\mathcal{C} \times \mathcal{D}}\left(U \times V, U^{\prime} \times V^{\prime}\right)=\operatorname{Hom}_{\mathcal{C}}(U, V) \times \operatorname{Hom}_{\mathcal{D}}\left(U^{\prime}, V^{\prime}\right)$ of morphism sets of $\mathcal{C}$ and $\mathcal{D}$. If $\mathcal{C}$ and $\mathcal{D}$ are monoidal, then so is $\mathcal{C} \times \mathcal{D}$, with tensor product $\otimes$ defined component-wise, i.e. as $(U \times V)$ $\otimes\left(U^{\prime} \times V^{\prime}\right)=\left(U \otimes_{\mathcal{C}} U^{\prime}\right) \times\left(V \otimes_{\mathcal{D}} V^{\prime}\right)$ on objects and analogously on morphisms and on associators.

For $\mathcal{C}=\mathcal{D}$ this product of categories turns out to have a family of module categories. These provide a categorification of the corresponding structure for rings (see the introduction). Accord-

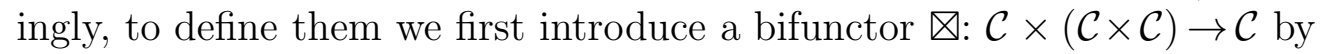

$$
M \otimes(U \times V):=M \otimes U \otimes V \quad \text { and } \quad m \otimes(f \times g):=m \otimes f \otimes g
$$

\footnotetext{
$\ddagger$ Following $\mathrm{OS}$, by strictness we mean that the $\gamma_{M, X}$ are isomorphisms. In the present paper all module functors are strict, so we drop this qualifier in the sequel.
} 

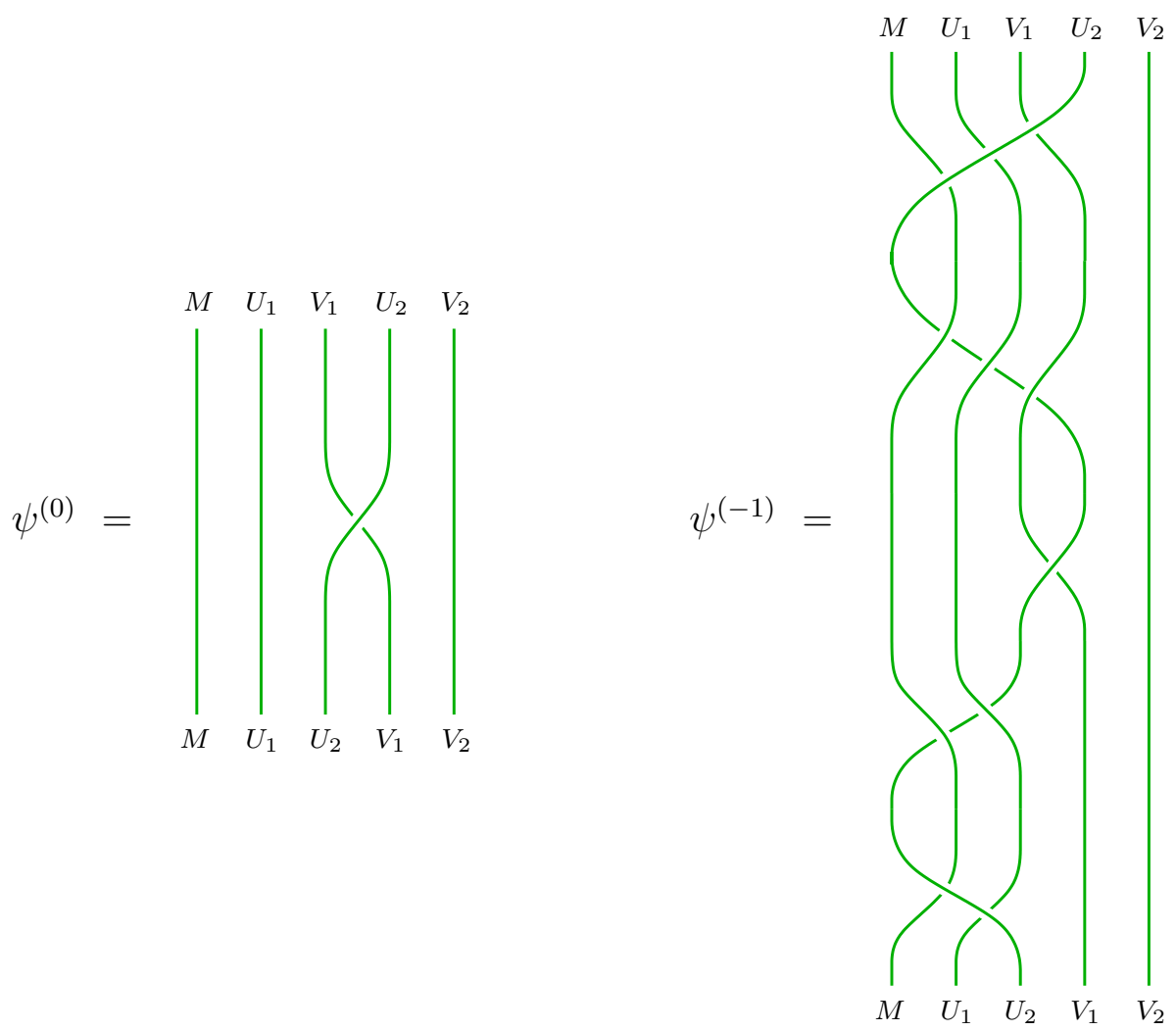

Figure 1: The morphism (2.9) for $n=0$ and $n=-1$, using the graphical calculus for morphisms in braided monoidal categories (see e.g. [BK, ERS1, [JS).

for objects $M$ in $\mathcal{C}$ and $U \times V$ in $\mathcal{C} \times \mathcal{C}$ and morphisms $m: M \rightarrow M^{\prime}$ and $f \times g: U \times V \rightarrow U^{\prime} \times V^{\prime}$. In order to obtain the structure of a module category on $\mathcal{C}$ we need more data, and to this end we have to require additional structure for $\mathcal{C}$, analogous to the commutativity requirement in the case of rings: $\mathcal{C}$ must be braided. We denote a braided monoidal category by $(\mathcal{C}, \otimes, \mathbf{1}, c)$, where $c$ is the braiding, i.e. a family of isomorphisms $c_{U, V}: U \otimes V \rightarrow V \otimes U$ satisfying the conditions of [JS, Def. 2.1]. We will use the shorthand

$$
D_{U, V}:=c_{V, U} \circ c_{U, V}: \quad U \otimes V \rightarrow U \otimes V
$$

for the two-fold braiding.

With these preparations, we can formulate

Theorem 2. Let $(\mathcal{C}, \otimes, \mathbf{1}, c)$ be a braided monoidal category. For every $n \in \mathbb{Z}, \mathcal{C}^{(n)} \equiv\left(\mathcal{C}, \bigotimes, \psi^{(n)}, r\right)$ with $\square$ as in (2.X), $r_{M}:=i d_{M}$ and

$$
\psi_{M, U_{1} \times V_{1}, U_{2} \times V_{2}}^{(n)}:=\left[\left(D_{M \otimes U_{1} \otimes V_{1}, U_{2}}\right)^{-n} \circ\left(i d_{M \otimes U_{1}} \otimes c_{U_{2}, V_{1}}\right) \circ\left[\left(D_{M \otimes U_{1}, U_{2}}\right)^{n} \otimes i d_{V_{1}}\right]\right] \otimes i d_{V_{2}}
$$

is a (right) module category over $\mathcal{C} \times \mathcal{C}$.

(For the pictorial representation of $\psi^{(n)}$ in the cases $n=0$ and $n=-1$ see figure 1)

Proof. Henceforth we will suppress the tensor product of $\mathcal{C}$ on objects, e.g. we will write $U V$ instead of $U \otimes V$. Naturality of $\psi^{(n)}$ follows from naturality of the braiding of $\mathcal{C}$, and the $\psi^{(n)}$ are 
clearly isomorphisms. The triangle identity of $\mathcal{C}^{(n)}$ holds trivially. It remains to check the pentagon identity. Substituting the definitions, the left hand side of (2.1) with $X=U_{1} \times V_{1}, Y=U_{2} \times V_{2}$ and $Z=U_{3} \times V_{3}$ reads

$$
\begin{aligned}
& \left\{\left[D_{M U_{1} V_{1} U_{2} V_{2}, U_{3}}^{-n} \circ\left(i d_{M U_{1} V_{1} U_{2}} \otimes c_{U_{3}, V_{2}}\right) \circ\left(D_{M U_{1} V_{1} U_{2}, U_{3}}^{n} \otimes i d_{V_{2}}\right)\right] \otimes i d_{V_{3}}\right\} \\
& \circ\left\{\left[D_{M U_{1} V_{1}, U_{2} U_{3}}^{-n} \circ\left(i d_{M U_{1}} \otimes c_{U_{2} U_{3}, V_{1}}\right) \circ\left(D_{M U_{1}, U_{2} U_{3}}^{n} \otimes i d_{V_{1}}\right)\right] \otimes i d_{V_{2} V_{3}}\right\},
\end{aligned}
$$

while for the right hand side of (2.1) one finds

$$
\begin{aligned}
& \left\{\left[D_{M U_{1} V_{1}, U_{2}}^{-n} \circ\left(i d_{M U_{1}} \otimes c_{U_{2}, V_{1}}\right) \circ\left(D_{M U_{1}, U_{2}}^{n} \otimes i d_{V_{1}}\right)\right] \otimes i d_{V_{2} U_{3} V_{3}}\right\} \\
& \quad \circ\left\{\left[D_{M U_{1} U_{2} V_{1} V_{2}, U_{3}}^{-n} \circ\left(i d_{M U_{1} U_{2}} \otimes c_{U_{3}, V_{1} V_{2}}\right) \circ\left(D_{M U_{1} U_{2}, U_{3}}^{n} \otimes i d_{V_{1} V_{2}}\right)\right] \otimes i d_{V_{3}}\right\} .
\end{aligned}
$$

Since $D_{U, V}$ is natural in both of its arguments we can move the morphism $D_{M U_{1} U_{2} V_{1} V_{2}, U_{3}}^{-n}$ in (2.11) all the way to the left, where it becomes $D_{M U_{1} V_{1} U_{2} V_{2}, U_{3}}^{-n}$, so that it can then be canceled against the corresponding morphism in (2.10). Next we rewrite the braiding $c_{U_{3}, V_{1} V_{2}}$ in (2.11) as $c_{U_{3}, V_{1} V_{2}}=\left(i d_{V_{1}} \otimes c_{U_{3}, V_{2}}\right) \circ\left(c_{U_{3}, V_{1}} \otimes i d_{V_{2}}\right)$. Then we move the braiding $c_{U_{3}, V_{2}}$ all the way to the left, so that we can cancel it against the corresponding braiding in (2.10). We may then also omit the common tensor factor $\mathrm{id}_{V_{2} V_{3}}$ from both sides. This shows that the pentagon is equivalent to

$$
\begin{array}{r}
D_{M U_{1} V_{1} U_{2}, U_{3}}^{n} \circ D_{M U_{1} V_{1}, U_{2} U_{3}}^{-n} \circ\left(i d_{M U_{1}} \otimes c_{U_{2} U_{3}, V_{1}}\right) \circ\left(D_{M U_{1}, U_{2} U_{3}}^{n} \otimes i d_{V_{1}}\right) \\
=\left(D_{M U_{1} V_{1}, U_{2}}^{-n} \otimes i d_{U_{3}}\right) \circ\left(i d_{M U_{1}} \otimes c_{U_{2}, V_{1}} \otimes i d_{U_{3}}\right) \circ\left(i d_{M U_{1} U_{2}} \otimes c_{U_{3}, V_{1}}\right) \\
\circ\left(D_{M U_{1}, U_{2}}^{n} \otimes i d_{U_{3} V_{1}}\right) \circ\left(D_{M U_{1} U_{2}, U_{3}}^{n} \otimes i d_{V_{1}}\right) .
\end{array}
$$

Now $D_{M U_{1}, U_{2}} \otimes \operatorname{id}_{U_{3}}$ and $D_{M U_{1} U_{2}, U_{3}}$ commute owing to naturality of $D_{U, V}$. We can therefore write $\left(D_{M U_{1}, U_{2}}^{n} \otimes i d_{U_{3}}\right) \circ D_{M U_{1} U_{2}, U_{3}}^{n}=\left(\left(D_{M U_{1}, U_{2}} \otimes i d_{U_{3}}\right) \circ D_{M U_{1} U_{2}, U_{3}}\right)^{n}$. Further, by direct calculation using the properties of the braiding one shows that

$$
\left(D_{M U_{1}, U_{2}} \otimes \mathrm{id}_{U_{3}}\right) \circ D_{M U_{1} U_{2}, U_{3}}=\left(i d_{M U_{1}} \otimes D_{U_{2}, U_{3}}\right) \circ D_{M U_{1}, U_{2} U_{3}}
$$

(it helps to draw the corresponding pictures, see figure 2). Again, the two terms on the right hand side of this equality commute, so that we can distribute the $n$th power over both terms, after which we can cancel $D_{M U_{1}, U_{2} U_{3}}^{n}$ against the corresponding term on the left hand side of (2.12). Because of $\left(c_{U_{2}, V_{1}} \otimes \mathrm{id}_{U_{3}}\right) \circ\left(\mathrm{id}_{U_{2}} \circ c_{U_{3}, V_{1}}\right)=c_{U_{2} U_{3}, V_{1}}$ and naturality of the braiding, the remaining braidings now cancel from (2.12). Bringing all terms involving negative powers to the opposite side of the equality and noting that the various factors commute thus allows us to rewrite (2.12) as

$$
\left[\left(D_{M U_{1} V_{1}, U_{2}} \otimes i d_{U_{3}}\right) \circ D_{M U_{1} V_{1} U_{2}, U_{3}}\right]^{n}=\left[\left(i d_{M U_{1} V_{1}} \otimes D_{U_{2}, U_{3}}\right) \circ D_{M U_{1} V_{1}, U_{2} U_{3}}\right]^{n}
$$

A short calculation using the properties of the braiding (again it helps to draw pictures) shows that the terms inside the two square brackets in the equality (2.14) are equal, and hence that (2.14) is indeed satisfied. This establishes the pentagon relation (2.1).

We mention that for $n=0$ and $n=1$ the associativity isomorphisms involve a single braiding:

$$
\psi_{M, U_{1} \times V_{1}, U_{2} \times V_{2}}^{(0)}=i d_{M U_{1}} \otimes c_{U_{2}, V_{1}} \otimes i d_{V_{2}}
$$




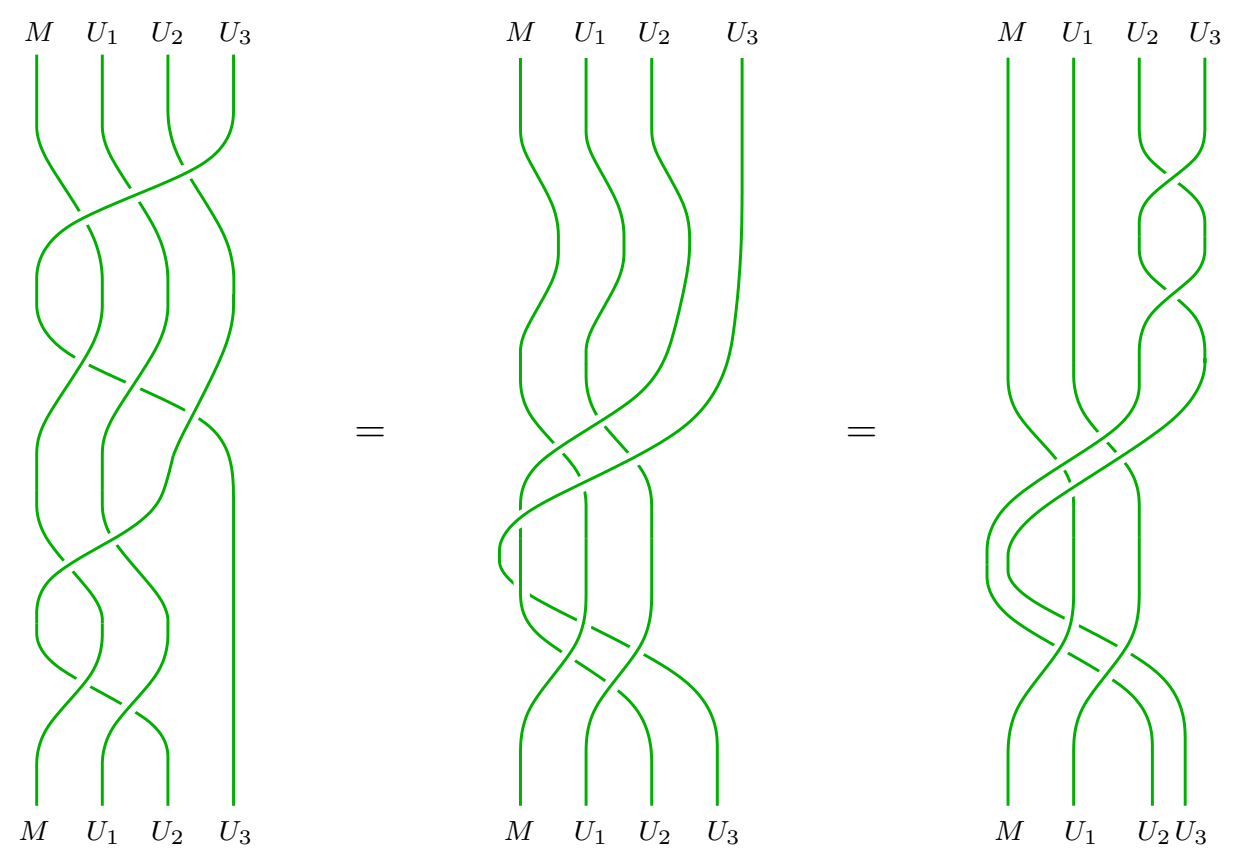

Figure 2: Proof of the equality (2.13).

and

$$
\psi_{M, U_{1} \times V_{1}, U_{2} \times V_{2}}^{(1)}=i d_{M U_{1}} \otimes c_{V_{1}, U_{2}}^{-1} \otimes i d_{V_{2}} .
$$

For other values of $n$ these isomorphisms are more complicated, as illustrated for $n=-1$ in figure 1 .

We also have

Corollary 3. For $(\mathcal{C}, \otimes, \mathbf{1}, c)$ a braided monoidal category and any $n \in \mathbb{Z},\left(\mathcal{C}, \widehat{\otimes}, \widehat{\psi}^{(n)}, l\right)$, with $\widehat{\otimes}:(\mathcal{C} \times \mathcal{C}) \times \mathcal{C} \rightarrow \mathcal{C}$ given by $(U \times V) \widehat{\otimes} M:=U \otimes V \otimes M$ on objects and analogously on morphisms, with $l_{M}:=i d_{M}$ and with

$\widehat{\psi}_{X, Y, M}^{(n)}:(X \otimes Y) \otimes M \rightarrow X \otimes(Y \otimes M)$ given by

$$
\widehat{\psi}_{U_{1} \times V_{1}, U_{2} \times V_{2}, M}^{(n)}:=i d_{U_{1}} \otimes\left[\left(D_{V_{1}, U_{2} \otimes V_{2} \otimes M}\right)^{n} \circ\left(c_{V_{1}, U_{2}}^{-1} \otimes i d_{V_{2} \otimes M}\right) \circ\left[i d_{U_{2}} \otimes\left(D_{V_{1}, V_{2} \otimes M}\right)^{-n}\right]\right],
$$

is a left module category over $\mathcal{C} \times \mathcal{C}$.

Proof. The proof is completely parallel to the one of Theorem 2. When expressing morphisms via the graphical tensor calculus (see figure 1), it amounts to a left-right reflection of the pictures for the proof of Theorem 2 .

In the sequel we concentrate on the right module category structure of $\mathcal{C}$. Obviously, all statements have an analogue for the left module category structure.

Additional structure on the braided monoidal category $\mathcal{C} \equiv(\mathcal{C}, \otimes, \mathbf{1}, c)$ can be used to relate the different module category structures described above. According to [JS, Def.6.1] a twist (or balancing) on $\mathcal{C}$ is a natural family $\theta$ of isomorphisms $\theta_{U}: U \rightarrow U$ such that $\theta_{\mathbf{1}}=i d_{\mathbf{1}}$ and

$$
D_{U, V}=\theta_{U V} \circ\left(\theta_{U}^{-1} \otimes \theta_{V}^{-1}\right)
$$

for all $U, V \in \mathcal{O b j}(\mathcal{C})$. 


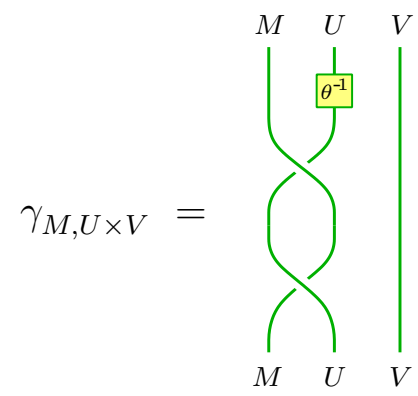

Figure 3: The morphism (2.19).

Theorem 4. Let $\mathcal{C}$ and $\mathcal{C}^{(n)}$ be as in Theorem 0 . The following are equivalent:

(i) For every $m, n \in \mathbb{Z}$ there exists a module functor $\left(i_{\mathcal{C}}, \gamma\right): \mathcal{C}^{(m)} \rightarrow \mathcal{C}^{(n)}$.

(ii) There is a module functor $\left(\mathrm{id}_{\mathcal{C}}, \gamma\right): \mathcal{C}^{(0)} \rightarrow \mathcal{C}^{(1)}$.

(iii) $\mathcal{C}$ can be endowed with a twist.

In particular, if $\mathcal{C}$ has a twist, then the module categories $\mathcal{C}^{(n)}$ defined in Theorem 2 are mutually equivalent. In theorem $\theta$ the statement (i) $\Rightarrow$ (ii) is trivial. The other statements are implied by the following two lemmas.

Lemma 5. Let $\theta$ be a twist on $\mathcal{C}$. Then $\left(\mathrm{id}_{\mathcal{C}}, \gamma\right): \mathcal{C}^{(n)} \rightarrow \mathcal{C}^{(n+1)}$ with

$$
\gamma_{M, U \times V}:=\left[\theta_{M \otimes U}^{-1} \circ\left(\theta_{M} \otimes i d_{U}\right)\right] \otimes i d_{V}
$$

for $M, U, V \in \mathcal{O} b j(\mathcal{C})$ is a module functor.

(See figure 3 for a pictorial representation of the morphism $\gamma_{M, U \times V}$ )

Proof. We must show that

$$
\left(\gamma_{M, X} \otimes i d_{Y}\right) \circ \gamma_{M \otimes X, Y} \circ \psi_{M, X, Y}^{(n)}=\psi_{M, X, Y}^{(n+1)} \circ \gamma_{M, X \otimes Y}
$$

for all $M \in \mathcal{O} b j(\mathcal{C})$ and $X, Y \in \mathcal{O} b j(C \times C)$. For $X=U_{1} \times V_{1}$ and $Y=U_{2} \times V_{2}$ this can be written as

$$
\begin{aligned}
\{ & {\left.\left[\theta_{M U_{1}}^{-1} \circ\left(\theta_{M} \otimes i d_{U_{1}}\right)\right] \otimes i d_{V_{1} U_{2} V_{2}}\right\} \circ\left\{\left[\theta_{M U_{1} V_{1} U_{2}}^{-1} \circ\left(\theta_{M U_{1} V_{1}} \otimes i d_{U_{2}}\right)\right] \otimes i d_{V_{2}}\right\} } \\
& \circ \psi_{M, U_{1} \times V_{1}, U_{2} \times V_{2}}^{(n)} \\
= & \left(D_{M U_{1} V_{1}, U_{2}}^{-1} \otimes i d_{V_{2}}\right) \circ \psi_{M, U_{1} \times V_{1}, U_{2} \times V_{2}}^{(n)} \circ\left(D_{M U_{1}, U_{2}} \otimes i d_{V_{1} V_{2}}\right) \\
& \circ\left[\theta_{M U_{1} U_{2}}^{-1} \circ\left(\theta_{M} \otimes i d_{U_{1} U_{2}}\right)\right] \otimes i d_{V_{1} V_{2}} .
\end{aligned}
$$

As an illustrative example, in figure 1 we give a graphical verification in the case $n=-1$. The general case can be proven as follows. We express the doubled braidings in terms of twists via (2.18) and use the naturality of $\theta$ and of $\psi^{(n)}$ to cancel various twist morphisms. Thereby the equality (2.21) can be reduced to

$$
\left(\theta_{M U_{1}}^{-1} \otimes i d_{V_{1} U_{2} V_{2}}\right) \circ \psi_{M, U_{1} \times V_{1}, U_{2} \times V_{2}}^{(n)}=\psi_{M, U_{1} \times V_{1}, U_{2} \times V_{2}}^{(n)} \circ\left(\theta_{M U_{1}}^{-1} \otimes i_{U_{2} V_{1} V_{2}}\right) .
$$

That the latter equality is indeed satisfied is easily verified by inspection of the explicit form of $\psi^{(n)}$. 

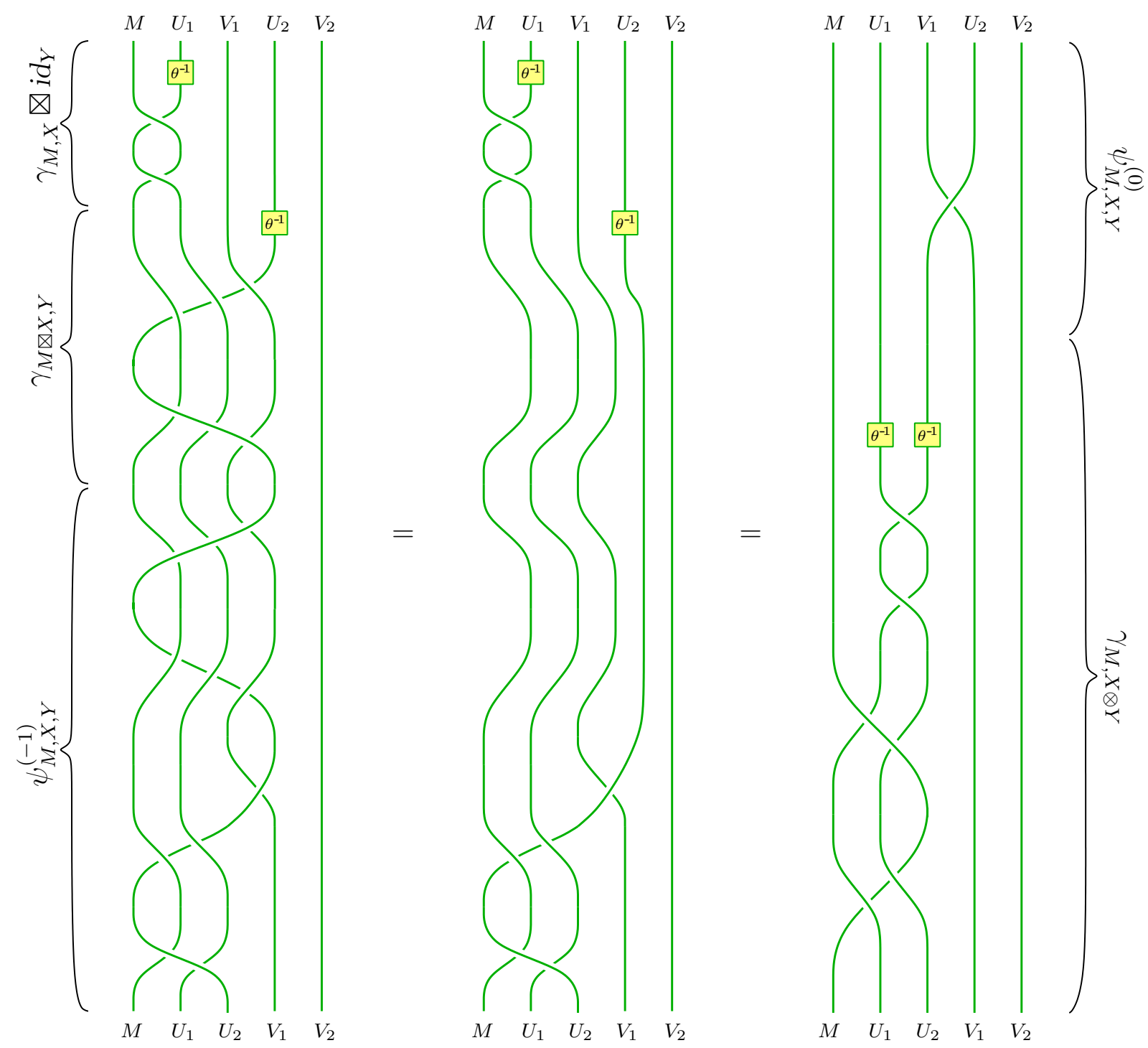

Figure 4: The equality (2.20) for $n=-1$.

Lemma 6. Let $\left(i d_{\mathcal{C}}, \gamma\right): \mathcal{C}^{(0)} \rightarrow \mathcal{C}^{(1)}$ be a module functor. Then

$$
\theta_{U}:=\gamma_{\mathbf{1}, \mathbf{1} \times U} \circ\left(\gamma_{\mathbf{1}, U \times \mathbf{1}}\right)^{-1}
$$

is a twist in $\mathcal{C}$.

Proof. By definition, $\theta_{\mathbf{1}}=i d_{\mathbf{1}}$, and naturality of $\theta$ follows from naturality of $\gamma$. Thus to establish that $\theta$ is a twist, it remains to verify the equality (2.18).

Using the explicit form (2.16) of $\psi^{(0)}$ and $\psi^{(1)}$, according to (2.20) we have

$$
\begin{aligned}
\left(\gamma_{M, U_{1} \times V_{1}} \otimes i d_{U_{2} V_{2}}\right) \circ \gamma_{M U_{1} V_{1}, U_{2} \times V_{2}} \circ\left(i d_{M U_{1}} \otimes c_{U_{2}, V_{1}} \otimes i d_{V_{2}}\right) & \\
= & \left(i d_{M U_{1}} \otimes c_{V_{1}, U_{2}}^{-1} \otimes i d_{V_{2}}\right) \circ \gamma_{M, U_{1} U_{2} \times V_{1} V_{2}} .
\end{aligned}
$$

When considered for $M=U_{2}=V_{1}=\mathbf{1}$ and $U_{1}=U, V_{2}=V$, this reduces to

$$
\left(\gamma_{\mathbf{1}, U \times \mathbf{1}} \otimes i d_{V}\right) \circ \gamma_{U, \mathbf{1} \times V}=\gamma_{\mathbf{1}, U \times V},
$$


and similarly one deduces that

$$
\left(\gamma_{M, \mathbf{1} \times U} \otimes i d_{V}\right) \circ \gamma_{M U, \mathbf{1} \times V}=\gamma_{M, \mathbf{1} \times U V}, \quad\left(\gamma_{\mathbf{1}, U \times \mathbf{1}} \otimes i d_{V}\right) \circ \gamma_{U, V \times \mathbf{1}}=\gamma_{\mathbf{1}, U V \times \mathbf{1}}
$$

and

$$
\left(\gamma_{\mathbf{1}, \mathbf{1} \times V} \otimes i d_{U}\right) \circ \gamma_{V, U \times \mathbf{1}} \circ c_{U, V}=c_{V, U}^{-1} \circ \gamma_{\mathbf{1}, U \times V} .
$$

When combined with (2.25) and (2.26), the relation (2.27) can be rewritten as

$$
\begin{aligned}
\left(\gamma_{\mathbf{1}, \mathbf{1} \times V} \otimes i d_{U}\right) \circ\left(\gamma_{\mathbf{1}, V \times \mathbf{1}}^{-1} \otimes i d_{U}\right) \circ \gamma_{\mathbf{1}, V U \times \mathbf{1}} \circ c_{U, V} \\
=c_{V, U}^{-1} \circ\left(\gamma_{\mathbf{1}, U \times \mathbf{1}} \otimes i d_{V}\right) \circ\left(\gamma_{\mathbf{1}, \mathbf{1} \times U}^{-1} \otimes i d_{V}\right) \circ \gamma_{\mathbf{1}, \mathbf{1} \times U V} .
\end{aligned}
$$

Next we note that naturality of $\gamma$ implies that

$$
\left(i d_{M} \otimes f \otimes g\right) \circ \gamma_{M, R_{1} \times S_{1}}=\gamma_{M, R_{2} \times S_{2}} \circ\left(i d_{M} \otimes f \otimes g\right)
$$

for all morphisms $f \times g: R_{1} \times S_{1} \rightarrow R_{2} \times S_{2}$ in $\mathcal{C} \times \mathcal{C}$. For $R_{1}=U V, R_{2}=V U, M=S_{1}=S_{2}=1$ and $f=c_{U, V}, g=i d_{1}$, this reads

$$
\gamma_{\mathbf{1}, V U \times \mathbf{1}} \circ c_{U, V}=c_{U, V} \circ \gamma_{\mathbf{1}, U V \times \mathbf{1}} .
$$

Combining this equality with $(2.28)$ and with the naturality of $c$, we finally obtain

$$
\left(\theta_{V} \otimes \theta_{U}\right) \circ c_{U, V}=c_{V, U}^{-1} \circ \theta_{U V}
$$

with $\theta$ as in (2.23). As a consequence, $\theta$ satisfies (2.18), as required.

Remark 7. In Theorem 1 we only consider module functors of the form $\left(i d_{\mathcal{C}}, \gamma\right): \mathcal{C}^{(m)} \rightarrow \mathcal{C}^{(n)}$. But in fact condition (i) of Theorem 1 can be replaced by the weaker requirement that for all $m, n \in \mathbb{Z}$ the module categories $\mathcal{C}^{(m)}$ and $\mathcal{C}^{(n)}$ are equivalent as module categories. To prove this, by Lemma 6 it is enough to show that the existence of an equivalence $(G, \gamma): \mathcal{C}^{(0)} \rightarrow \mathcal{C}^{(1)}$ implies the existence of an equivalence $($ id, $\tilde{\gamma}): \mathcal{C}^{(0)} \rightarrow \mathcal{C}^{(1)}$.

For $Y \in \mathcal{O} b j(\mathcal{C})$ denote by $T_{Y}$ the functor $Y \otimes-: \mathcal{C} \rightarrow \mathcal{C}$. One checks that $\left(T_{Y}, i d\right): \mathcal{C}^{(0)} \rightarrow \mathcal{C}^{(0)}$ is a module functor. Next one notes that $\gamma_{\mathbf{1}, U \times \mathbf{1}}: G(U) \rightarrow G(\mathbf{1}) \otimes U$ is a natural isomorphism of functors from $G$ to $T_{G(\mathbf{1})}$. Since $G$ is an equivalence of module categories, there exists a functor $(F, \varphi)$ such that $(G, \gamma) \circ(F, \varphi)$ is naturally isomorphic to $\left(\mathrm{id}_{\mathcal{C}}, \mathrm{id}\right)$ as a module functor. The composition $(G, \gamma) \circ\left(T_{F(\mathbf{1})}, i d\right)$ is then naturally isomorphic to $\left(i d_{\mathcal{C}}, \tilde{\gamma}\right)$ for some $\tilde{\gamma}$.

\section{Braided induction}

For the construction of the module category structures of $\mathcal{M}=\mathcal{C}$ over $\mathcal{X}=\mathcal{C} \times \mathcal{C}$ in the previous section one must assume that $\mathcal{C}$ is braided. From this assumption it follows that $\mathcal{X}$ can be endowed with a braiding, too:

$$
c_{U \times V, U^{\prime} \times V^{\prime}}^{\mathcal{C} \times \mathcal{C}}=c_{U, U^{\prime}} \times c_{V, V^{\prime}},
$$

where $c$ is the braiding of $\mathcal{X}$. Now it is known that to any module category over a braided category $\mathcal{X}$ there are associated two functors, called braided induction, from $\mathcal{X}$ to the category of module endofunctors. These turn out to be of interest also in the present context.

\footnotetext{
$\S$ In fact there are several braidings, depending on whether $c$ or $c^{-1}$ is taken in each factor. For us the choice $c \times c$ is the relevant one.
} 
If $(\mathcal{M}, \bigotimes, \psi)$ is a module category over a braided monoidal category $\mathcal{X}$, then for any $X \in \mathcal{O} b j(\mathcal{X})$ one defines [O] two module endofunctors $\alpha_{X}^{ \pm}$of $\mathcal{M}$ as follows. As functors they coincide, both acting as

$$
\alpha_{X}^{ \pm}(M):=M \otimes X \quad \text { and } \quad \alpha_{X}^{ \pm}(f):=f \otimes i d_{X}
$$

on objects and morphisms of $\mathcal{M}$, while their module functor structures $\gamma^{X, \pm}$ are given by

$$
\begin{aligned}
\gamma_{M, Y}^{X,+} & :=\psi_{M, X, Y} \circ\left(\mathrm{id}_{M} \otimes c_{Y, X}\right) \circ \psi_{M, Y, X}^{-1} \quad \text { and } \\
\gamma_{M, Y}^{X,-} & :=\psi_{M, X, Y} \circ\left(\mathrm{id}_{M} \otimes c_{X, Y}^{-1}\right) \circ \psi_{M, Y, X}^{-1},
\end{aligned}
$$

respectively.

For the module categories constructed in Theorem 2, it is straightforward to specify natural transformations between the endofunctors $\alpha^{ \pm}$- these will be instrumental in the proof of Proposition 18 below. Only the case $n=0$ of Theorem 2 will be needed for this purpose.

Lemma 8. Let $\mathcal{C}^{(0)}$ be the module category over $\mathcal{C} \times \mathcal{C}$ given in Theorem $⿴$. Then for any pair of objects $U, V \in \mathcal{O} b j(\mathcal{C})$, the collection

$$
\Gamma_{M}:=\left[\left(c_{V, M} \circ c_{M, V}\right) \otimes \mathrm{id}_{U}\right] \circ\left(\mathrm{id}_{M} \otimes c_{U, V}\right)
$$

of morphisms in $\operatorname{Hom}_{\mathcal{C}}(M \otimes U \otimes V, M \otimes V \otimes U)$ furnishes a natural isomorphism from $\alpha_{U \times V}^{+}$to $\alpha_{V \times U}^{-}$as module functors.

Proof. First note that $\alpha_{U \times V}^{ \pm}(M)=M \otimes U \otimes V$, so that the morphisms (3.4) belong to the correct morphism spaces. In view of the definition (3.2) of $\alpha_{X}^{ \pm}$and formulas (2.16) and (3.1) for the associator and the braiding, it is then obvious that these isomorphisms constitute a natural transformation from $\alpha_{U \times V}^{+}$to $\alpha_{V \times U}^{-}$as functors.

It thus remains to be checked that this natural transformation is compatible with the module functor property. This means that the equality

$$
\gamma_{M, U^{\prime} \times V^{\prime}}^{V \times U,-} \circ \Gamma_{M \otimes\left(U^{\prime} \times V^{\prime}\right)}=\left(\Gamma_{M} \otimes i d_{U^{\prime} \otimes V^{\prime}}\right) \circ \gamma_{M, U^{\prime} \times V^{\prime}}^{U \times V,+},
$$

with morphisms $\gamma^{X, \pm}$ as in (3.3), holds for all $M \in \mathcal{O} b j(\mathcal{C})$ and $U^{\prime} \times V^{\prime} \in \mathcal{O} b j(\mathcal{C} \times \mathcal{C})$. Now according to (2.16) and (3.1), not only the $\Gamma_{M}$, but also the relevant morphisms $\gamma^{X, \pm}$ are entirely expressible through the braiding of $\mathcal{C}$ :

$$
\begin{aligned}
& \gamma_{M, U^{\prime} \times V^{\prime}}^{U \times V,+}=i d_{M} \otimes\left[\left(i d_{U} \otimes c_{U^{\prime}, V} \otimes i d_{V^{\prime}}\right) \circ\left(c_{U^{\prime}, U} \otimes c_{V^{\prime}, V}\right) \circ\left(i d_{U^{\prime}} \otimes c_{U, V^{\prime}}^{-1} \circ i d_{V}\right)\right], \\
& \gamma_{M, U^{\prime} \times V^{\prime}}^{V \times U,}=i d_{M} \otimes\left[\left(i d_{V} \otimes c_{U^{\prime}, U} \otimes i d_{V^{\prime}}\right) \circ\left(c_{V, U^{\prime}}^{-1} \otimes c_{U, V^{\prime}}^{-1}\right) \circ\left(i d_{U^{\prime}} \otimes c_{V, V^{\prime}}^{-1} \circ i d_{U}\right)\right] .
\end{aligned}
$$

It is then an easy application of the properties of the braiding (amounting here to using relations in the braid group on five strands) to check that the equalities (3.5) are indeed satisfied.

The composition of functors endows the category $\mathcal{E} n d_{\mathcal{X}}(\mathcal{M})$ of module endofunctors with the structure of a strict monoidal category. (Also, $\mathcal{X}$ is a left module category over $\mathcal{E} n d_{\mathcal{X}}(\mathcal{M})$.) The functors $\alpha^{ \pm}$of braided induction (or $\alpha$-induction $[\mathrm{LR}, \mathrm{Xu},[\mathrm{BE}]$ ) are the two functors from $\mathcal{X}$ to $\mathcal{E} n d_{\mathcal{X}}(\mathcal{M})$ that act as

$$
\alpha^{ \pm}: \quad \mathcal{O} b j(\mathcal{X}) \ni X \longmapsto \alpha_{X}^{ \pm}
$$

on objects. One checks that $\alpha^{ \pm}$together with the associativity and unit constraints of $\mathcal{M}$ constitute monoidal functors from $\left(\mathcal{X}, \otimes^{\text {opp }}\right)$ to $\mathcal{E} n d_{\mathcal{X}}(\mathcal{M})$. The braided induction functors are of 
particular interest in the case that $\mathcal{X}$ is a modular tensor category and a few other conditions (see [FRS3, Thm O]) on the module category are satisfied. In that situation every module endofunctor of $\mathcal{M}$ can be obtained as a retract, in the monoidal category $\mathcal{E} n d_{\mathcal{X}}(\mathcal{M})$, of one of the objects $\alpha_{X}^{+} \circ \alpha_{Y}^{-} \in \mathcal{E} n d_{\mathcal{X}}(\mathcal{M})$, see Theorem 4.10 of [FFRS]. Crucial information about the module category $\mathcal{M}$ over $\mathcal{X}$ is then encoded in the spaces $\operatorname{Hom}_{\mathcal{E}_{n} d_{\mathcal{X}}(\mathcal{M})}\left(\alpha_{X}^{+}, \alpha_{Y}^{-}\right)$of natural transformations between braided induction functors.

\section{Completion with respect to direct sums}

In the sequel we will be interested in the case that $\mathcal{C}$ is premodular with ground field $\mathbb{C}$, that is $[\mathrm{Br}]$, an abelian $\mathbb{C}$-linear semisimple category with finitely many isomorphism classes of simple objects, endowed with a ribbon structure (i.e. being braided monoidal and having a compatible twist and dualities, see e.g. [Ka]), and with simple monoidal unit. Then the tensor product of $\mathcal{C}$ is exact and thus preserves direct sums. As a consequence, also the bifunctor $\square$ defined as in (2.7) preserves direct sums.

Braided induction is compatible with direct sums as well. Indeed, it is straightforward to check that, for $\mathcal{M}$ a module category over $\mathcal{X}$ and $\left\{X_{\ell}\right\}$ any family of objects in $\mathcal{X}$ whose direct sum $\bigoplus_{\ell} X_{\ell}$ exists, for every $M \in \mathcal{O} b j(\mathcal{M})$ the object $\alpha_{\oplus_{\ell} X_{\ell}}^{ \pm}(M)$ of $\mathcal{M}$ satisfies the universal property of the direct sum $\bigoplus_{\ell}\left(\alpha_{X_{\ell}}^{ \pm}(M)\right)$.

In the case of premodular categories the following notion of product of categories is relevant.

Definition 9. Let $\mathcal{C}$ be an abelian $\mathbb{C}$-linear category. Then the category $\mathcal{C} \otimes \mathcal{C}$ is defined as follows. $\mathcal{O} b j(\mathcal{C} \otimes \mathcal{C})$ is obtained by completing the class of pairs $U \times V$ of objects of $\mathcal{C}$ with respect to direct sums. The morphism sets of $\mathcal{C} \otimes \mathcal{C}$ are tensor products $\operatorname{Hom}_{\mathcal{C} \otimes \mathcal{C}}\left(U \times V, U^{\prime} \times V^{\prime}\right)=$ $\operatorname{Hom}_{\mathcal{C}}(U, V) \otimes_{\mathbb{C}} \operatorname{Hom}_{\mathcal{C}}\left(U^{\prime}, V^{\prime}\right)$ of morphism spaces of $\mathcal{C}$, extended to direct sums.

If $\mathcal{C}$ is premodular, then so is $\mathcal{C} \otimes \mathcal{C}$, with tensor product (denoted by $\otimes$ again) and braiding (denoted by $c$ again) defined in the same way as in the case of $\mathcal{C} \times \mathcal{C}$, extended to direct sums. As a consequence, from Theorem 2 we can deduce that for $\mathcal{C}$ a $\mathbb{C}$-linear braided monoidal category, for any $n \in \mathbb{Z}, \mathcal{C}^{(n)} \equiv\left(\mathcal{C}, \bigotimes, \psi^{(n)}\right)$ with

$$
\otimes: \quad M \times\left(\bigoplus_{\ell} U_{\ell} \times V_{\ell}\right) \mapsto \bigoplus_{\ell} M \otimes U_{\ell} \otimes V_{\ell}
$$

and

$$
\begin{aligned}
\psi_{M, \oplus_{l}\left(U_{l} \times V_{l}\right), \oplus_{m}\left(U_{m} \times V_{m}\right)}^{(n)} & :=\bigoplus_{l, m}\left[\left(D_{M U_{l} V_{l}, U_{m}}\right)^{-n} \circ\left(i d_{M U_{l}} \otimes c_{U_{m}, V_{l}}\right) \circ\left[\left(D_{M U_{l}, U_{m}}\right)^{n} \otimes i d_{V_{l}}\right]\right] \otimes i d_{V_{m}}
\end{aligned}
$$

is a (right) module category over $\mathcal{C} \otimes \mathcal{C}$.

Similarly, from Theorem 4 and Remark 7 we learn that the following statements about $\left(\mathcal{C}, \mathbb{\otimes}, \psi^{(n)}\right)$ are equivalent:

(i) For every $m, n \in \mathbb{Z}$ there exists a module functor $\left(i_{\mathcal{C}}, \gamma\right): \mathcal{C}^{(m)} \rightarrow \mathcal{C}^{(n)}$.

(ii) $\mathcal{C}$ can be endowed with a twist.

$\left(i^{\prime}\right)$ For every $m, n \in \mathbb{Z}$ the module categories $\mathcal{C}^{(m)}$ and $\mathcal{C}^{(n)}$ are equivalent. 
Convention 10. In the sequel, unless stated otherwise, the category $\mathcal{C}$ will be assumed to be a premodular category. We choose a set $\left\{U_{i} \mid i \in I\right\}$ of representatives of the simple objects of $\mathcal{C}$, and we set $N_{i j}{ }^{k}:=\operatorname{dim}_{\mathbb{C}}\left(\operatorname{Hom}_{\mathcal{C}}\left(U_{i} \otimes U_{j}, U_{k}\right)\right)$.

\section{A family of algebras in $\mathcal{C} \otimes \mathcal{C}$}

Recall from [OS, Thm. 1] that any semisimple indecomposable (right) module category $\mathcal{M}$ over a semisimple rigid monoidal category $\mathcal{X}$ with finitely many isomorphism classes of simple objects and with simple unit object is equivalent to the category of (left) $A$-modules for some algebra $A$ in $\mathcal{X}$. Such an algebra can be obtained as the internal End $\underline{\operatorname{End}}(M)$ of a non-zero object $M$ in $\mathcal{M}$; different objects of $\mathcal{M}$ lead to Morita equivalent algebras.

First recall from [OS, Sec.3.2] that for two objects $M_{1}$ and $M_{2}$ of $\mathcal{M}$ their internal Hom $\underline{\operatorname{Hom}}\left(M_{1}, M_{2}\right)$ is an object of $\mathcal{X}$ that represents the functor

$$
\begin{aligned}
\mathcal{X} & \rightarrow \text { Vect }_{\mathbb{k}} \\
X & \mapsto \operatorname{Hom}_{\mathcal{M}}\left(M_{1} \otimes X, M_{2}\right) .
\end{aligned}
$$

The internal Hom always exists, when $\mathcal{X}$ and $\mathcal{M}$ are semisimple and $\mathcal{X}$ has only finitely many isomorphism classes of simple objects. For $M=M_{1}=M_{2}$ we write $\underline{\operatorname{End}}(M):=\underline{\operatorname{Hom}}(M, M)$ and call this object the internal End of $M$. There is a canonical evaluation morphism $e_{M_{1}, M_{2}}$ : $M_{1} \otimes \underline{\operatorname{Hom}}\left(M_{1}, M_{2}\right) \rightarrow M_{2}$ in $\mathcal{M}$ that induces an associative composition morphism

$$
\underline{\operatorname{Hom}}\left(M_{1}, M_{2}\right) \otimes \underline{\operatorname{Hom}}\left(M_{2}, M_{3}\right) \rightarrow \underline{\operatorname{Hom}}\left(M_{1}, M_{3}\right) .
$$

This morphism gives the structure of an associative algebra on the internal End of an object $M$ in $\mathcal{M}$.

In the situation at hand there is a distinguished object in $\mathcal{M}$, namely the tensor unit $\mathbf{1}$. We are interested in the algebra structure of the internal End of $\mathbf{1}$. The multiplication morphism depends on which of the module category structures $\mathcal{C}^{(n)}$ of section to we choose; accordingly we denote the internal End of $\mathbf{1}$ by $\underline{\operatorname{End}}^{(n)}(\mathbf{1})$. In the sequel we will again use the graphical calculus for morphisms, now for the case of premodular categories. We depict the morphisms in dual bases $\left\{f_{\alpha}\right\}$ of $\operatorname{Hom}_{\mathcal{C}}\left(U_{i} \otimes U_{j}, U_{k}\right)$ and $\left\{\bar{f}_{\alpha}\right\}$ of $\operatorname{Hom}_{\mathcal{C}}\left(U_{k}, U_{i} \otimes U_{j}\right)$ as

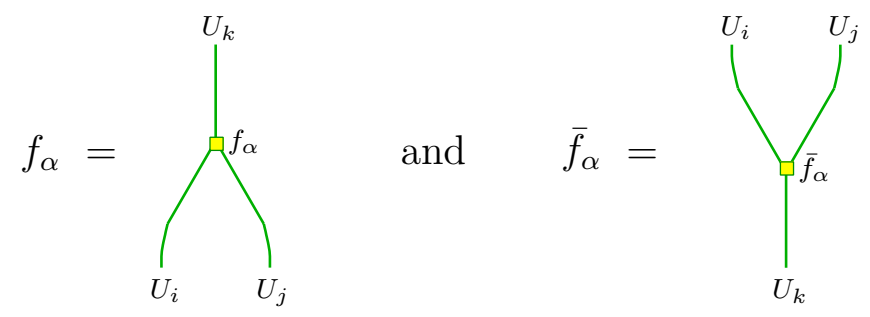

Theorem 11. Let $\mathcal{C}$ and $\mathcal{C}^{(n)}$ be as in section 母. As an object of $\mathcal{C} \otimes \mathcal{C}$,

$$
\underline{\operatorname{End}}^{(n)}(\mathbf{1}) \cong \bigoplus_{i \in \mathcal{I}} U_{i}^{\vee} \times U_{i}=: A
$$


The multiplication of the algebra $\underline{\text { End }}^{(n)}(\mathbf{1})=\left(A, m^{(n)}, \eta\right)$ is given by

$$
m^{(n)}=\bigoplus_{i, j, k \in \mathcal{I}} \sum_{\alpha=1}^{N_{i j}^{k}}
$$

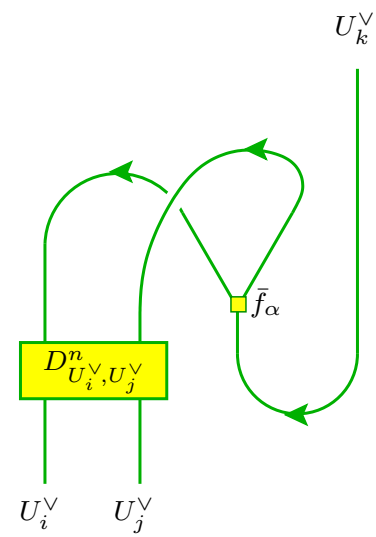

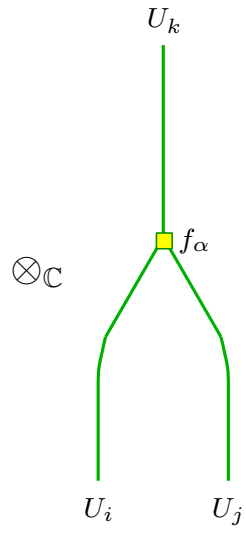

The unit morphism is $\eta=e_{\mathbf{1} \times \mathbf{1} \prec A}$, the embedding morphism of $\mathbf{1} \times \mathbf{1}$ as a subobject of $A$.

For $n=0$ this algebra has been studied, in different contexts, in [M̈̈1, FFRS, KR1]. Observe that the product $m^{(n)}$ is independent of the choice of bases of the morphism spaces $\operatorname{Hom}_{\mathcal{C}}\left(U_{i} \otimes U_{j}, U_{k}\right)$.

Proof. We perform the following steps:

(1) Determine the structure of an internal End on the object $A$.

(2) Find the evaluation morphism $\mathrm{OS} e v_{\mathbf{1}, \mathbf{1}}: \mathbf{1} \otimes A \rightarrow \mathbf{1}$.

(3) Compute the multiplication morphism on $A$.

Only the last step involves the different structures of module categories on $\mathcal{C}$.

(1) We consider the functors

$$
\begin{aligned}
& T: \quad \mathcal{C} \otimes \mathcal{C} \rightarrow \mathcal{C}, \quad \bigoplus_{\ell} V_{\ell} \times W_{\ell} \mapsto \bigoplus_{\ell} V_{\ell} \otimes W_{\ell} \\
& \bigoplus_{\ell} f_{\ell} \otimes_{\mathbb{C}} g_{\ell} \mapsto \bigoplus_{\ell} f_{\ell} \otimes g_{\ell} \\
& \text { and } \quad R: \quad \mathcal{C} \rightarrow \mathcal{C} \otimes \mathcal{C}, \quad M \mapsto \bigoplus_{i \in \mathcal{I}}\left(M \otimes U_{i}^{\vee}\right) \times U_{i} \text {, } \\
& f \mapsto \bigoplus_{i \in \mathcal{I}}\left(f \otimes i d_{U_{i}}\right) \otimes_{\mathbb{C}} i d_{U_{i}} .
\end{aligned}
$$

As shown in [KR2], $R$ is a right adjoint for $T$, with the adjunction isomorphisms

$$
\phi_{X}^{M}: \quad \operatorname{Hom}_{\mathcal{C}}(T(X), M) \stackrel{\cong}{\rightarrow} \operatorname{Hom}_{\mathcal{C} \otimes \mathcal{C}}(X, R(M))
$$

given by

$$
\phi_{X}^{M}: f=\bigoplus_{\ell} f_{\ell} \longmapsto \bigoplus_{\ell} \bigoplus_{i \in \mathcal{I}} \sum_{\beta}\left[\left(\left[f_{\ell} \circ\left(i d_{V_{\ell}} \otimes g_{\beta}\right)\right] \otimes i d_{U_{i}^{\vee}}\right) \circ\left(i d_{V_{\ell}} \otimes b_{U_{i}}\right)\right] \otimes_{\mathbb{C}} \bar{g}_{\bar{\beta}}
$$

for $X=\bigoplus_{\ell} V_{\ell} \times W_{\ell}$, where $f_{\ell} \in \operatorname{Hom}_{\mathcal{C}}\left(V_{\ell} \otimes W_{\ell}, M\right)$ and the $\beta$-summation is over a basis $\left\{g_{\beta}\right\}$ of $\operatorname{Hom}_{\mathcal{C}}\left(U_{i}, W_{\ell}\right)$ and the dual basis $\left\{\bar{g}_{\bar{\beta}}\right\}$ of $\operatorname{Hom}_{\mathcal{C}}\left(W_{\ell}, U_{i}\right)$. Note that again this is independent of the choice of a basis.

Now observe that $T(X)=\mathbf{1} \otimes X$ and $R(\mathbf{1})=A$. Thus for $M=\mathbf{1}$ the prescription (5.7) furnishes an isomorphism

$$
\phi_{X}^{\mathbf{1}}: \quad \operatorname{Hom}_{\mathcal{C}}(\mathbf{1} \otimes X, \mathbf{1}) \stackrel{\cong}{\rightarrow} \operatorname{Hom}_{\mathcal{C} \otimes \mathcal{C}}(X, A) .
$$

This shows that $A$ possesses the defining property of $\underline{\text { End }}(\mathbf{1})$. Henceforth we abbreviate $\phi_{X} \equiv \phi_{X}^{\mathbf{1}}$. 
(2) By $\mathrm{OS}$, the evaluation morphism $e v_{\mathbf{1}, \mathbf{1}} \in \operatorname{Hom}_{\mathcal{C}}(\mathbf{1} \otimes A, \mathbf{1})$ is obtained as $e v_{\mathbf{1 , 1}}=\phi_{A}^{-1}\left(\mathrm{id}_{A}\right)$. Now we have

$$
\phi_{A}\left(\bigoplus_{j \in \mathcal{I}} d_{U_{j}}\right)=\bigoplus_{i, j \in \mathcal{I}} \sum_{\beta}\left[\left(\left[d_{U_{j}} \circ\left(i d_{U_{j} \vee} \otimes g_{\beta}\right)\right] \otimes i d_{U_{i} \vee}\right) \circ\left(i d_{U_{j} \vee} \otimes b_{U_{i}}\right)\right] \otimes_{\mathbb{C}} \bar{g}_{\beta}
$$

with $\left\{g_{\beta}\right\}$ a basis of $\operatorname{Hom}_{\mathcal{C}}\left(U_{i}, U_{j}\right)$. Since $\operatorname{Hom}_{\mathcal{C}}\left(U_{i}, U_{j}\right) \cong \delta_{i, j} \mathbb{C}$, only $i=j$ contributes to the $i$ summation. Choosing $g_{\beta}=i d_{U_{i}}$ we get

$$
\phi_{A}\left(\bigoplus_{j \in \mathcal{I}} d_{U_{j}}\right)=\bigoplus_{i \in \mathcal{I}}\left[\left(d_{U_{i}} \circ \otimes i d_{U_{i} \vee}\right) \circ\left(i d_{U_{i}} \otimes b_{U_{i}}\right)\right] \otimes_{\mathbb{C}} i d_{U_{i}}=\bigoplus_{i \in \mathcal{I}} i d_{U_{i} \vee} \otimes_{\mathbb{C}} i d_{U_{i}}=i d_{A}
$$

Thus $e v_{\mathbf{1}, \mathbf{1}}=\phi_{A}^{-1}\left(i d_{A}\right)=\bigoplus_{j \in \mathcal{I}} d_{U_{j}}$.

(3) The multiplication on $\underline{\text { End }}^{(n)}(\mathbf{1})$ is obtained as the image of $e v_{\mathbf{1}, \mathbf{1}} \circ\left(e v_{\mathbf{1}, \mathbf{1}} \otimes i d_{A}\right) \circ \psi_{\mathbf{1}, A, A}^{(n)}$ under $\phi_{A \otimes A}$. We compute

$$
\begin{aligned}
& e v_{\mathbf{1}, \mathbf{1}} \circ\left(e v_{\mathbf{1}, \mathbf{1}} \otimes i d_{A}\right) \circ \psi_{\mathbf{1}, A, A}^{(n)} \\
& =\bigoplus_{i, j \in \mathcal{I}}\left(d_{U_{i}} \otimes d_{U_{j}}\right) \circ\left(D_{U_{i}^{\vee} U_{i}, U_{j} \vee}^{-n} \otimes i d_{U_{j}}\right) \circ \\
& \left(i d_{U_{i} \vee} \otimes c_{U_{j} \vee U_{i}} \otimes i d_{U_{j}}\right) \circ\left(D_{U_{i}^{\vee}, U_{j} \vee}^{n} \otimes i d_{U_{i} U_{j}}\right) \\
& =\bigoplus_{i, j \in \mathcal{I}} d_{U_{j}} \circ\left(D_{1, U_{j} \vee}^{-n} \otimes i d_{U_{j}}\right) \circ\left(d_{U_{i}} \otimes i d_{U_{j} \vee U_{j}}\right) \circ \\
& \left(i d_{U_{i} \vee} \otimes c_{U_{j} \vee U_{i}} \otimes i d_{U_{j}}\right) \circ\left(D_{U_{i}^{\vee}, U_{j} \vee}^{n} \otimes i d_{U_{i} U_{j}}\right) \\
& =\bigoplus_{i, j \in \mathcal{I}}\left(d_{U_{i}} \otimes d_{U_{j}}\right) \circ\left(i d_{U_{i} \vee} \otimes c_{U_{j} \vee U_{i}} \otimes i d_{U_{j}}\right) \circ\left(D_{U_{i}^{\vee}, U_{j} \vee}^{n} \otimes i d_{U_{i} U_{j}}\right)
\end{aligned}
$$

Here the second equality uses naturality of $D_{U, V}$, and the third one that $D_{1, U}=i d_{U}$. We then find that, in terms of the dual bases $\left\{f_{\alpha}\right\}$ and $\left\{\bar{f}_{\alpha}\right\}$ in (5.3),

$$
\begin{aligned}
m^{(n)}= & \phi_{A \otimes A}\left(e v_{\mathbf{1}, \mathbf{1}} \circ\left(e v_{\mathbf{1}, \mathbf{1}} \otimes i d_{A}\right) \circ \psi_{\mathbf{1}, A, A}^{(n)}\right) \\
= & \bigoplus_{i, j, k \in \mathcal{I}} \sum_{\alpha}\left[\left(\left[\left(d_{U_{i}} \otimes d_{U_{j}}\right) \circ\left(i d_{U_{i} \vee} \otimes c_{U_{j}^{\vee}, U_{i}} \otimes i d_{U_{j}}\right) \circ\left(D_{U_{i}^{\vee}, U_{j} \vee}^{n} \otimes i d_{U_{i} U_{j}}\right)\right.\right.\right. \\
& \left.\left.\left.\quad \circ\left(i d_{U_{i} \vee U_{j} \vee} \otimes \bar{f}_{\alpha}\right)\right] \otimes i d_{U_{k} \vee}\right) \circ\left(i d_{U_{i} \vee U_{j} \vee} \otimes b_{U_{k}}\right)\right] \otimes_{\mathbb{C}} f_{\alpha} .
\end{aligned}
$$

This is indeed the morphism given in (5.5).

Since the module categories $\left(\mathcal{C}, \otimes, \psi^{(n)}\right)$ are mutually equivalent, all the algebras $\left(A, m^{(n)}, \eta\right)$ are Morita equivalent. But in fact we even have

Lemma 12. All algebra structures $\left(A, m^{(n)}, \eta\right)$ with $n \in \mathbb{Z}$ are isomorphic.

Proof. The morphism

$$
\sigma:=\bigoplus_{i \in \mathcal{I}}\left(\theta_{U_{i}} \vee \otimes_{\mathbb{C}} i d_{U_{i}}\right)
$$

is an automorphism of $A$ as an object. As an easy consequence of naturality and the defining property 2.18$)$ of the twist, $\sigma$ indeed constitutes an algebra isomorphism from $\left(A, m^{(n)}, \eta\right)$ to $\left(A, m^{(n+1)}, \eta\right)$.

It follows from the proof of Lemma 12 that we can express $m^{(n)}$ entirely through $m^{(0)}$ and the twist:

$$
m^{(n)}=\sigma^{n} \circ m^{(0)} \circ\left(\sigma^{-n} \otimes \sigma^{-n}\right)
$$

with $\sigma$ as given in (5.14). 


\section{$6 \quad$ Frobenius algebras}

That the object $A=\underline{\text { End }}(\mathbf{1})$ has the structure of an algebra is guaranteed by the results of OS]. But in fact $A$ carries more structure, namely the one of a symmetric special Frobenius algebra. A Frobenius algebra in a monoidal category $\mathcal{X}$ is an algebra in $\mathcal{X}$ that is also a coalgebra, with the coproduct being a morphism of $A$-bimodules. For $\mathcal{X}$ sovereign, a symmetric Frobenius algebra $B=(B, m, \eta, \Delta, \varepsilon)$ in $\mathcal{X}$ is a Frobenius algebra for which the two isomorphisms $\left((\varepsilon \circ m) \otimes i d_{B \vee}\right) \circ\left(i d_{B} \otimes b_{B}\right)$ and $\left(i d_{B} \vee \otimes(\varepsilon \circ m)\right) \circ\left(\tilde{b}_{B} \otimes i d_{B}\right)$ from $B$ to its dual $B^{\vee}$ are equal, and a symmetric special Frobenius algebra is a symmetric Frobenius algebra for which $m \circ \Delta=i d_{B}$ and $\varepsilon \circ \eta=\operatorname{dim}(B)$ id $_{\mathbf{1}}$ with $\operatorname{dim}(B) \neq 0$.

The global dimension $\operatorname{Dim}(\mathcal{C})$ of $\mathcal{C}$ is the sum of the squares of dimensions of its simple objects,

$$
\operatorname{Dim}(\mathcal{C}):=\sum_{i \in \mathcal{I}} \operatorname{dim}\left(U_{i}\right)^{2}
$$

By ENO, Thm. 2.3\& Cor 2.10], $\operatorname{dim}\left(U_{i}\right)$ is real and non-zero, and $\operatorname{Dim}(\mathcal{C}) \geq 1$.

Proposition 13. (1) $\left(A, m^{(n)}, \eta, \Delta^{(n)}, \varepsilon\right)$ with $m^{(n)}$ and $\eta$ as defined in Theorem 11 and with

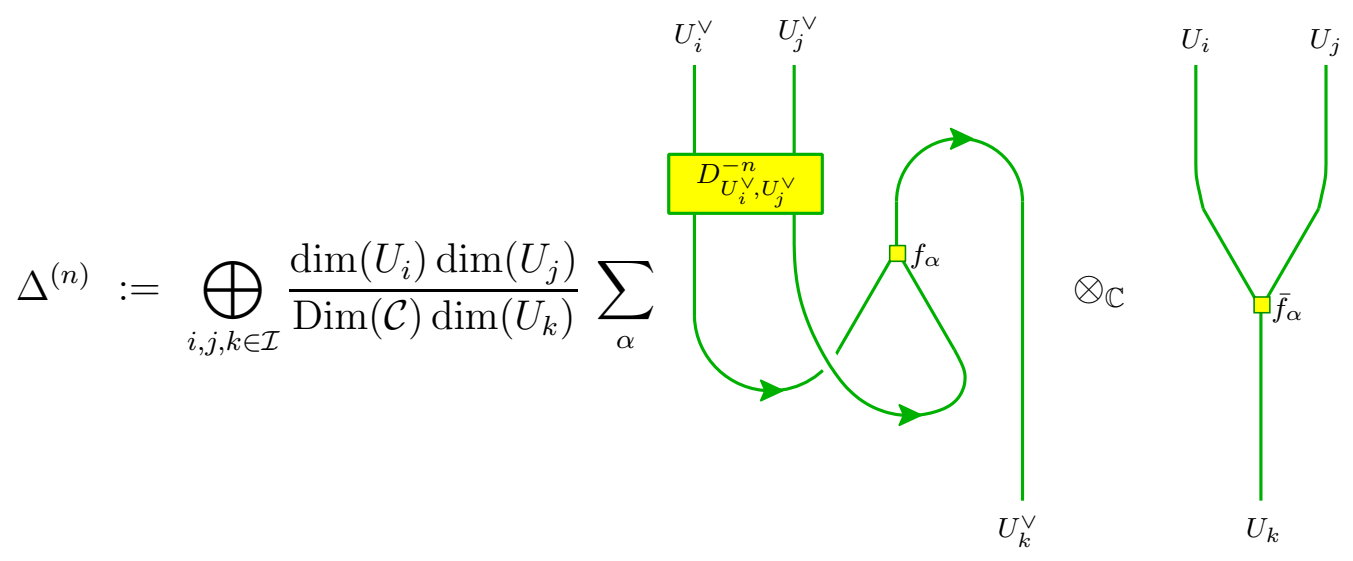

(with the $\alpha$-summation as in (5.5)) and

$$
\varepsilon:=\operatorname{Dim}(\mathcal{C}) r_{A \succ \mathbf{1} \times \mathbf{1}}
$$

(with $r_{A \succ \mathbf{1} \times \mathbf{1}}$ the restriction morphism for $\mathbf{1} \times \mathbf{1}$ as a subobject of $A$ ) is a symmetric special Frobenius algebra.

(2) The algebras $\left(A, m^{(n)}, \eta, \Delta^{(n)}, \varepsilon\right)$, with $n \in \mathbb{Z}$, are all isomorphic as Frobenius algebras.

Proof. (1) For every $n$ consider the morphism

$$
\begin{aligned}
\Phi^{(n)} & :=\left(d_{A} \otimes i d_{A^{\vee}}\right) \circ\left(i d_{A} \otimes m^{(n)} \otimes i d_{A^{\vee}}\right) \circ\left(\tilde{b}_{A} \otimes m^{(n)} \otimes i d_{A^{\vee}}\right) \circ\left(i d_{A} \otimes b_{A}\right) \\
& \in \operatorname{Hom}\left(A, A^{\vee}\right) .
\end{aligned}
$$

Starting from the definition of $m^{(n)}$ and using $D_{U, \mathbf{1}}=i d_{U}$ and $d_{U} \circ D_{U^{\vee}, U}=d_{U} \circ\left(\theta_{U^{\vee}}^{2} \otimes i d_{U}\right)$, it is 
straightforward to obtain the explicit expression

$$
\Phi^{(n)}=\operatorname{Dim}(\mathcal{C}) \bigoplus_{i \in \mathcal{I}} \frac{1}{\operatorname{dim}\left(U_{i}\right)}
$$
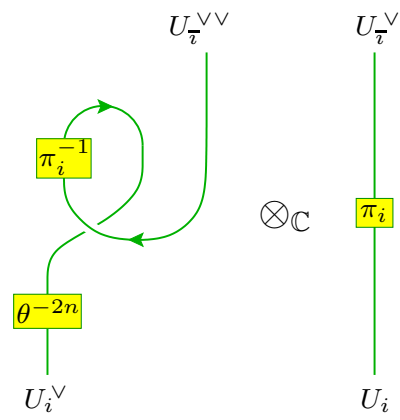

for these morphisms, where $\theta \equiv \theta_{U_{i}^{\vee}}, \bar{k} \in \mathcal{I}$ is the label for which $U_{\bar{k}} \cong U_{k}^{\vee}$, and $\pi_{k} \in \operatorname{Hom}_{\mathcal{C}}\left(U_{k}, U_{\bar{k}}^{\vee}\right)$ is a choice of isomorphisms for each $k \in \mathcal{I}$ such that the compatibility condition (2.24) of [ERS1] between $\pi_{k}$ and $\pi_{\bar{k}}$ is satisfied. This shows in particular that $\Phi^{(n)}$ is invertible.

Now according to Lemma 3.12 of FRS1 invertibility of $\Phi^{(n)}$ implies that $\left(A, m^{(n)}, \eta\right)$ can be endowed with the structure of a symmetric special Frobenius algebra, with the counit and coproduct given by $\varepsilon^{(n)}=d_{A} \circ\left(\Phi^{(n)} \otimes \eta\right)=d_{A} \circ\left(i d_{A} \otimes m^{(n)}\right) \circ\left(\tilde{b}_{A} \otimes i d_{A}\right)$ and by

$$
\begin{aligned}
\Delta^{(n)} & =\left(m^{(n)} \otimes \Phi^{(n)^{-1}}\right) \circ\left(i_{A} \otimes b_{A}\right) \\
& =\left(d_{A} \otimes \Phi^{(n)^{-1}} \otimes \Phi^{(n)^{-1}}\right) \circ\left(i_{A} \otimes m^{(n)} \otimes i d_{A} \otimes i d_{A}\right) \circ\left(\Phi^{(n)} \otimes b_{A \otimes A}\right),
\end{aligned}
$$

respectively. Again by direct calculation one shows that these are precisely the morphisms given in the proposition.

(2) Two Frobenius algebras are isomorphic as Frobenius algebras if and only if via one and the same isomorphism they are isomorphic both as algebras and as coalgebras. That all the algebras $\left(A, m^{(n)}, \eta, \Delta^{(n)}, \varepsilon\right)$ are isomorphic as algebras, with an isomorphism given by the appropriate power of $\sigma$ (5.14), has already been established in Lemma 12. Given the explicit form of the coproduct and counit, an entirely analogous argument shows that these morphisms constitute isomorphisms of coalgebras as well.

Note that we can write $\Phi^{(n)}=\Phi^{(0)} \circ \sigma^{-2 n}$ and

$$
\Delta^{(n)}=\left(\sigma^{n} \otimes \sigma^{n}\right) \circ \Delta^{(0)} \circ \sigma^{-n}
$$

with $\sigma$ as in (5.14). The coproduct $\Delta^{(0)}$ already appeared in [KR2, (2.57)].

\section{Azumaya algebras}

A modular tensor category $\mathcal{X}$ is a premodular category for which the symmetric $|\mathcal{I}| \times|\mathcal{I}|$-matrix $S$ with entries

$$
S_{i j}=S_{00}\left(d_{X_{j}} \otimes \tilde{d}_{X_{i}}\right) \circ\left[i d_{X_{i}^{\vee}} \otimes\left(c_{X_{i}, X_{j}} \circ c_{X_{j}, X_{i}}\right) \otimes i d_{X_{j}^{\vee}}\right] \circ\left(\tilde{b}_{X_{j}} \otimes b_{X_{i}}\right)
$$

is invertible; here $\left\{X_{i} \mid i \in \mathcal{I}\right\}$ denotes a set of representatives for the isomorphism classes of simple objects of $\mathcal{X}$, and $S_{00}=(\operatorname{Dim}(\mathcal{C}))^{-1 / 2}>0$.

If the category $\mathcal{C}$ in Convention 10 is even modular, then, as we will see, the special symmetric Frobenius algebra $A$ from Proposition 13 has an additional property: it is Azumaya. 
Definition 14. An Azumaya category $\mathcal{M}$ over a braided monoidal category $\mathcal{X}$ is a (right) module category $\mathcal{M}$ over $\mathcal{X}$ for which the two monoidal functors $\alpha^{ \pm}(3.7)$ from $\left(\mathcal{X}, \otimes^{\text {opp }}\right)$ to $\mathcal{E} n d_{\mathcal{X}}(\mathcal{M})$ are equivalences.

An Azumaya algebra $B$ in a braided monoidal category $\mathcal{X}$ is an algebra $B$ in $\mathcal{X}$ such that the category of (left) $B$-modules is an Azumaya category over $\mathcal{X}$.

For $B$ a symmetric special Frobenius algebra in a modular tensor category $\mathcal{X}$ and $\mathcal{X}_{B}$ the category of left $B$-modules in $\mathcal{X}$, the endofunctor category $\mathcal{E} n d_{\mathcal{X}}\left(\mathcal{X}_{B}\right)$ is monoidally equivalent to the category $\mathcal{X}_{B \mid B}$ of $B$-bimodules in $\mathcal{X}$. As a consequence, in this case the definition of Azumaya algebra given here is equivalent to the one given in $\mathrm{VZ}$.

For $B$ a symmetric special Frobenius algebra in a modular tensor category, consider the endomorphism

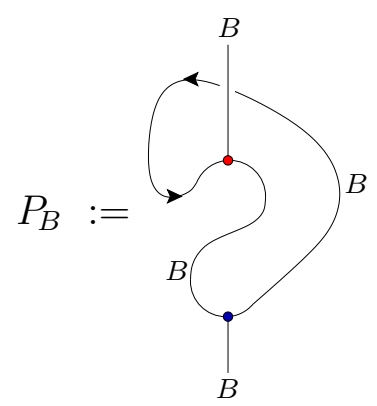

which (e.g. by Lemma 5.2 of [FRS1]) is an idempotent.

Lemma 15. A symmetric special Frobenius algebra $B$ in a modular tensor category $\mathcal{X}$ is Azumaya if and only if

$$
P_{B}=\frac{1}{\operatorname{dim}(B)} \eta \circ \varepsilon
$$

Proof. By [FFRS, Prop. 2.30] the image of the idempotent $P_{B}$ of $B$ is the left center $C_{l}(B)$ as defined in FFRS, Def. 2.31]. Because of $\varepsilon \circ \eta=\operatorname{dim}(B) i d_{1}$, the equality (7.3) is therefore equivalent to the statement that $C_{l}(B) \cong \mathbf{1}$. Further, according to Proposition 2.36 of [FFRS] one has

$$
\operatorname{Hom}_{\mathcal{X}}\left(C_{l}(B) \otimes X, Y\right) \cong \operatorname{Hom}_{\mathcal{E} n d_{\mathcal{X}}(\mathcal{M})}\left(\alpha_{X}^{+}, \alpha_{Y}^{+}\right) .
$$

As we will now show, it follows that the braided induction functors $\alpha^{ \pm}$from $\mathcal{X}$ to $\mathcal{E} n d_{\mathcal{X}}(\mathcal{M})$ are equivalences iff $C_{l}(B)$ is trivial.

(i) Suppose that $\alpha^{+}$is an equivalence of categories. Then by $(7.4)$ we have $\operatorname{Hom}_{\mathcal{X}}\left(C_{l}(B) \otimes X, Y\right)$ $\cong \operatorname{Hom}_{\mathcal{X}}(X, Y)$ for all $X, Y \in \mathcal{O} b j(\mathcal{X})$, which implies that $C_{l}(B) \cong \mathbf{1}$.

(ii) Suppose that $C_{l}(B) \cong \mathbf{1}$. Then owing to the isomorphism (7.4) $\alpha^{+}$is bijective on morphisms. That $\alpha^{+}$is also essentially surjective on objects, and hence an equivalence, is seen as follows. Again by (7.4), $\alpha_{X_{i}}^{+}$with $i \in \mathcal{I}$ are mutually non-isomorphic simple objects of $\mathcal{E}_{n} d_{\mathcal{X}}(\mathcal{M})$. The same holds for $\left\{\alpha_{X_{i}}^{-} \mid i \in \mathcal{I}\right\}$. As a consequence, each row of the $|\mathcal{I}| \times|\mathcal{I}|$-matrix with entries $Z_{i j}:=\operatorname{dim}_{\mathbb{C}}\left(\operatorname{Hom}_{\mathcal{E n d}_{\mathcal{X}}(\mathcal{M})}\left(\alpha_{X_{i}}^{+}, \alpha_{X_{j}}^{-}\right)\right.$contains at most one non-zero entry, and this non-zero entry, if present, must be equal to 1 . It follows that $n:=\sum_{i, j \in \mathcal{I}}\left(Z_{i j}\right)^{2} \leq|\mathcal{I}|$. On the other hand, according to [FRS1, Sect. 5.4\& Rem. 5.19], $n$ is equal to the number of isomorphism classes of simple objects in $\mathcal{E} n d_{\mathcal{X}}(\mathcal{M})$. Since the $\alpha_{X_{i}}^{+}$already provide $|\mathcal{I}|$ distinct simple objects, we must have $n=|\mathcal{I}|$. Thus in particular $\alpha^{+}$is essentially surjective, as claimed.

The reasoning that $\alpha^{-}$is an equivalence is analogous. 
Proposition 16. If $\mathcal{C}$ is a modular tensor category, then the algebra $\left(A, m^{(n)}, \eta\right)$ introduced in Theorem 11 is Azumaya.

Proof. For $A$ as defined above, we have $\operatorname{dim}(A)=\operatorname{Dim}(\mathcal{C})$ and $(7.3)$ reads $P_{A}=e_{\mathbf{1} \times \mathbf{1} \prec A} \circ r_{A \succ \mathbf{1} \times \mathbf{1}}$, which may be rewritten as $P_{A}=\bigoplus_{i \in \mathcal{I}} \delta_{i, 0} i d_{U_{i}^{\vee} \times U_{i}}$. Because of Lemma 12 it suffices to establish this equality for the case $n=0$.

Inserting the explicit form of the product and coproduct into (7.2), and rewriting the resulting morphisms in the first factor of $\mathcal{C} \otimes \mathcal{C}$ with the help of the properties of the braiding (which amount to simple deformations of the graphical representation of the morphism), one shows that

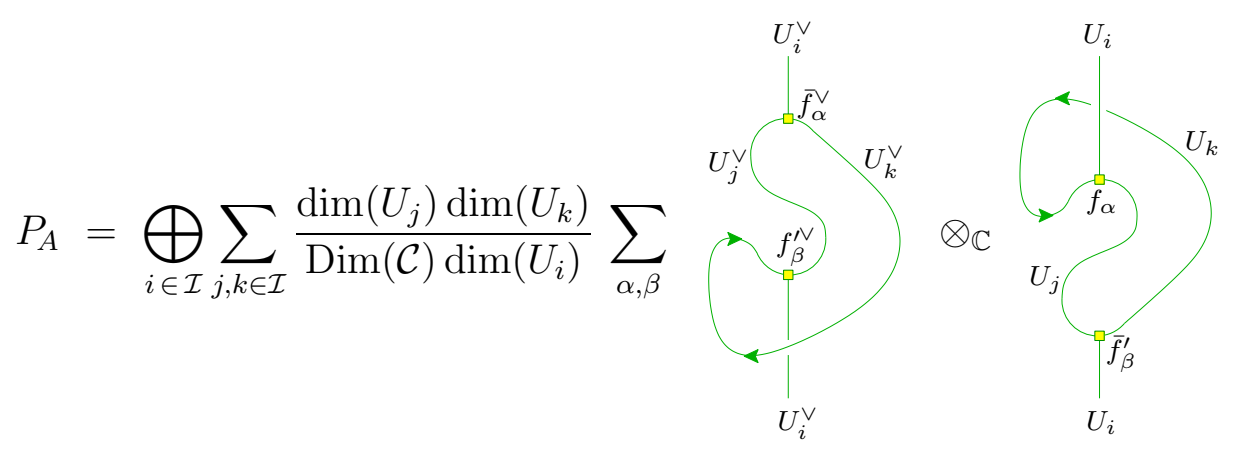

where the $\alpha$-summation is over a basis $\left\{f_{\alpha}\right\}$ of $\operatorname{Hom}_{\mathcal{C}}\left(U_{k} \otimes U_{j}, U_{i}\right)$ and the corresponding dual basis of $\operatorname{Hom}_{\mathcal{C}}\left(U_{i}, U_{k} \otimes U_{j}\right)$, and similarly for the $\beta$-summation. Next we note that the morphisms $u_{i ; \alpha, \beta}^{(j, k)} \in \operatorname{End}_{\mathcal{C}}\left(U_{i}^{\vee}\right)$ and $v_{i ; \alpha, \beta}^{(j, k)} \in \operatorname{End}_{\mathcal{C}}\left(U_{i}\right)$ that are represented by the two diagrams on the right hand side of this equality are multiples of $i d_{U_{i}} \vee$ and of $i d_{U_{i}}$, respectively. As a consequence, using properties of dual bases one can write

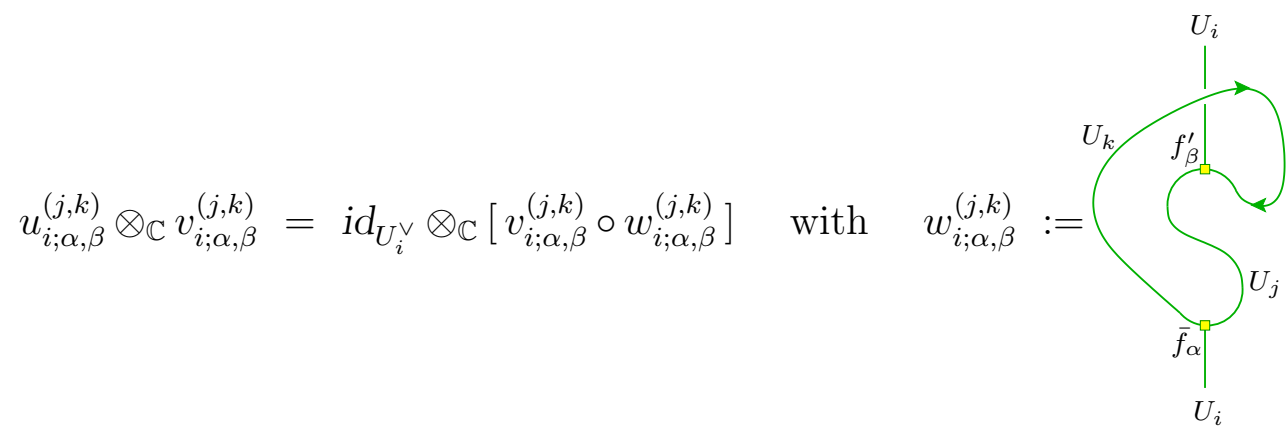

Performing the $\beta$-summation leads to

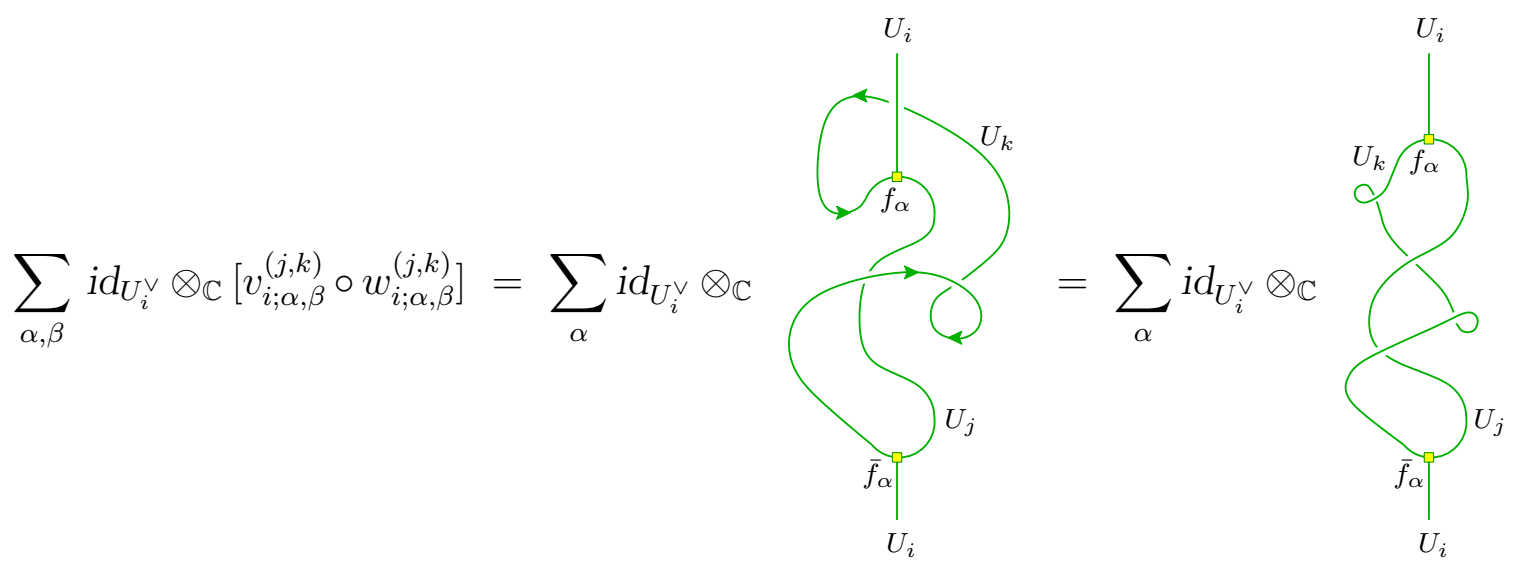


Using the naturality of the twist and the fact that the twist of a simple object is a multiple of the identity morphism, $\theta_{U_{l}}=\vartheta_{l} i d_{U_{l}}$, the second factor on the right hand side of (7.7) can be rewritten as

$$
\begin{aligned}
f_{\alpha} \circ c_{U_{j}, U_{k}} \circ c_{U_{k}, U_{j}} \circ\left(\theta_{U_{k}}^{2} \otimes\right. & \left.\mathrm{id}_{U_{j}}\right) \circ \bar{f}_{\alpha}=\frac{\vartheta_{k}}{\vartheta_{j}} f_{\alpha} \circ c_{U_{j}, U_{k}} \circ c_{U_{k}, U_{j}} \circ\left(\theta_{U_{k}} \otimes \theta_{U_{j}}\right) \circ \bar{f}_{\alpha} \\
& =\frac{\vartheta_{k}}{\vartheta_{j}} f_{\alpha} \circ \theta_{U_{k} \otimes U_{j}} \circ \bar{f}_{\alpha}=\frac{\vartheta_{k}}{\vartheta_{j}} f_{\alpha} \circ \bar{f}_{\alpha} \circ \theta_{U_{i}}=\frac{\vartheta_{k} \vartheta_{i}}{\vartheta_{j}} i d_{U_{i}} .
\end{aligned}
$$

After these manipulations the $\alpha$-summation has become trivial, yielding just the dimension $N_{k j}^{i}$ of the morphism space $\operatorname{Hom}_{\mathcal{C}}\left(U_{k} \otimes U_{j}, U_{i}\right)$. Summarising the calculations performed so far, we thus find that

$$
P_{A}=\bigoplus_{i \in \mathcal{I}} \xi_{i} i_{U_{i}^{\vee}} \otimes_{\mathbb{C}} i d_{U_{i}} \quad \text { with } \quad \xi_{i}=\sum_{j, k \in \mathcal{I}} \frac{\operatorname{dim}\left(U_{j}\right) \operatorname{dim}\left(U_{k}\right)}{\operatorname{Dim}(\mathcal{C}) \operatorname{dim}\left(U_{i}\right)} \frac{\vartheta_{i} \vartheta_{k}}{\vartheta_{j}} N_{k j}^{i}
$$

Now since $\mathcal{C}$ is not only premodular, but even modular, then (see e.g. [BK, Ch. 3.1]) the dimensions $N_{i j}{ }^{k}$ can be expressed through the $|\mathcal{I}| \times|\mathcal{I}|$-matrix $S$ as $N_{i j}{ }^{k}=\sum_{l \in \mathcal{I}} S_{i l} S_{j l} S_{l k}^{*} / S_{0 l}$, and the matrices $S$ and $t$ with $t_{i j}=\vartheta_{i} \delta_{i, j}$ furnish a projective representation of the group $\mathrm{SL}(2, \mathbb{Z})$. In particular we have $S t S=\gamma t^{-1} S t^{-1} C$ and $S t^{-1} S=\gamma^{-1} t S t$, where $\gamma \in \mathbb{C}^{\times}$is a constant and $C$, the charge conjugation matrix, is the matrix with entries $C_{k, l}=\delta_{k, \bar{l}}$. Together with $\operatorname{dim}\left(U_{i}\right)=S_{0 i} / S_{00}$, this allows us to rewrite the numbers $\xi_{i}$ as

$$
\begin{aligned}
\xi_{i} & =S_{00} \sum_{l \in \mathcal{I}} \frac{\vartheta_{i} S_{l i}^{*}}{S_{0 i} S_{0 l}}(S t S)_{0 l}\left(S t^{-1} S\right)_{0 l} \\
& =S_{00} \sum_{l \in \mathcal{I}} \frac{\vartheta_{i} S_{l i}^{*}}{S_{0 i} S_{0 l}}\left(t^{-1} S t^{-1}\right)_{0 l}(t S t)_{0 l}=S_{00} \frac{\vartheta_{i}}{S_{0 i}} \sum_{l \in \mathcal{I}} S_{i l}^{*} S_{l 0}=\delta_{i, 0},
\end{aligned}
$$

so that (7.9) reduces to $P_{A}=\bigoplus_{i \in \mathcal{I}} \delta_{i, 0} i d_{U_{i}^{\vee}} \otimes_{\mathbb{C}} i d_{U_{i}}$. Thus indeed $A$ is Azumaya.

Remark 17. The product (5.5) and coproduct (6.2) of $A$ do not make reference to the braiding in the second factor of $\mathcal{C} \otimes \mathcal{C}$. Denote by $\overline{\mathcal{C}}$ the modular tensor category obtained from $\mathcal{C}$ by replacing the braiding $c$ and the twist $\theta$ by their inverses. Then $A$ is also a symmetric special Frobenius algebra in $\mathcal{C} \otimes \overline{\mathcal{C}}$ (this is the situation considered in [Mü1, FFRS, [KR1]). As an algebra in $\mathcal{C} \nabla \overline{\mathcal{C}}$, $A$ is commutative, i.e. $C_{l}(A)=A=C_{r}(A)$; in this sense it is 'maximally non-Azumaya'.

As an important consequence of the Azumaya property of $A$ we have

Proposition 18. The spaces $\operatorname{Hom}_{\mathcal{E} n d_{\mathcal{C} \bowtie \mathcal{C}}(\mathcal{C})}\left(\alpha_{U_{i} \times U_{j}}^{+}, \alpha_{U_{k} \times U_{l}}^{-}\right)$have dimension

$$
\operatorname{dim}_{\mathbb{C}}\left(\operatorname{Hom}_{\mathcal{E n d}_{\mathcal{C} \otimes \mathcal{C}}(\mathcal{C})}\left(\alpha_{U_{i} \times U_{j}}^{+}, \alpha_{U_{k} \times U_{l}}^{-}\right)\right)=\delta_{i, l} \delta_{j, k},
$$

for all $i, j, k, l \in \mathcal{I}$.

Proof. Since $\mathcal{C}$ is equivalent to the category of $A$-modules in $\mathcal{C} \otimes \mathcal{C}$, the fact that $A$ is Azumaya implies [FRS2 that the $|\mathcal{I}|^{2} \times|\mathcal{I}|^{2}$-matrix $Z=\left(Z_{i j, k l}\right)$ with $Z_{i j, k l}=\operatorname{dim}_{\mathbb{C}}\left(\operatorname{Hom}_{\mathcal{E} n d_{\mathcal{C} \bowtie \mathcal{C}}(\mathcal{C})}\left(\alpha_{U_{i} \times U_{j}}^{+}, \alpha_{U_{k} \times U_{l}}^{-}\right)\right)$ is a permutation matrix, i.e. for any pair $(i, j) \in \mathcal{I} \times \mathcal{I}$ there is precisely one pair $(k, l)$ such that $Z_{i j, k l}=1$, while all other entries of $Z$ are zero. Now according to Lemma 8 , for any $i, j \in \mathcal{I}$ the morphism space $\operatorname{Hom}_{\mathcal{E n d}_{\mathcal{C} \bowtie \mathcal{C}}(\mathcal{C})}\left(\alpha_{U_{i} \times U_{j}}^{+}, \alpha_{U_{i} \times U_{j}}^{-}\right)$is non-zero, i.e. $Z_{i j, j i}=1$. 


\section{Permutation modular invariants}

We now address the application of our results to two-dimensional rational conformal quantum field theory (CFT), to which we already alluded in the introduction.

A full local conformal field theory can be constructed from two ingredients: a chiral conformal field theory, and one additional datum. A chiral conformal field theory is, by definition, the following mathematical object: a system of conformal blocks, i.e. sheaves over the moduli spaces of curves with marked points, with a Knizhnik-Zamolodchikov connection, constructed (see e.g. [FB, Fr]) from a conformal vertex algebra. In the case of our interest, the vertex algebra $\mathcal{V}$ is rational, which amounts [Hu] to the statement that its representation category $\mathcal{C}$ is a modular tensor category.

The additional datum needed for obtaining a full conformal field theory is then a (Morita class of symmetric special Frobenius algebra $(\mathrm{s}) A$ in the representation category $\mathcal{C}$. The set of such Morita classes is in bijection to the set of consistent collections of correlation functions defining a full local rational conformal field theory (see [FjFRS2, KR2]).

To interpret the results of the preceding sections in this context, suppose that the modular tensor category $\mathcal{C}$ considered there is the representation category of a rational vertex algebra $\mathcal{V}$. The monoidal category $\mathcal{C} \otimes \mathcal{C}$ is then equivalent to the representation category of the vertex algebra $\mathcal{V} \otimes_{\mathbb{C}} \mathcal{V}$. Further, the non-negative integers $Z_{i j, k l}(A)=\operatorname{dim}_{\mathbb{C}}\left(\operatorname{Hom}_{\mathcal{E} n d_{\mathcal{C} \otimes \mathcal{C}}(\mathcal{C})}\left(\alpha_{i, j}^{+}, \alpha_{k, l}^{-}\right)\right)$are then the coefficients, in the standard basis of characters, of the torus partition function of the full CFT associated to the algebra $A$ [FRS1]. It follows from Theorem 5.1 of [FRS1] that this matrix obeys all requirements on a so-called modular invariant, as formulated e.g. in Section V of [Ga].

It should be appreciated, though, that not every quadratic matrix with non-negative entries that obeys the axioms for a modular invariant describes the partition function of a full local conformal field theory. Counter examples even occur for the modular invariant induced by the duality on $\mathcal{C}$ [SS]. In view of this fact, the following application of the preceding results is noteworthy. Take $A$ to be the symmetric special Frobenius algebra given in Proposition 13. Then the result (7.11) amounts to the statement that the torus partition function of the full CFT determined by $A$ is given by the so-called $\mathbb{Z}_{2}$-permutation modular invariant. We thus arrive at

Corollary 19. The transposition modular invariant for a rational conformal field theory with chiral symmetry $\mathcal{V} \otimes_{\mathbb{C}} \mathcal{V}$ is physical, i.e. there exists a consistent full local conformal field theory with this modular invariant as its torus partition function (and it can be obtained via the construction of [FRS1]).

Remark 20. A word of warning is, however, in order: We do not have, at present, a classification of module categories over $\mathcal{C} \otimes \mathcal{C}$ yielding the transposition modular invariant for the partition function, i.e. we cannot exclude that there exist symmetric special Frobenius algebras not Morita equivalent to the algebra $A$ considered here that give the same torus partition function as $A$.

Remark 21. Proceeding along the lines of ERS1 one can compute other correlation functions of the full CFT associated to the algebra $A$. One of them is the partition function on an annulus. The coefficients of this correlator in a standard basis are called the annulus coefficients. As shown in [FRS1, these are given by the integers $\mathrm{A}_{X, M}:=\operatorname{dim}_{\mathbb{C}}\left(\operatorname{Hom}_{A}(M \otimes X, N)\right)$ with $X$ a simple object and $M, N$ simple $A$-modules. For the algebra $A$ considered here, the induced $A$-modules $M_{i}:=\operatorname{Ind}_{A}\left(1 \times U_{i}\right)$ with $i \in \mathcal{I}$ provide a complete set of pairwise nonisomorphic simple $A$-modules,

and a general induced $A$-module decomposes according to $\operatorname{Ind}_{A}\left(U_{i} \times U_{j}\right) \cong \bigoplus_{k \in \mathcal{I}} N_{i j}^{k} M_{k}($ compare 
Lemma 6.20 of [FFRS]). As a consequence, the annulus coefficients are

$$
\mathrm{A}_{U_{i} \times U_{j}, M_{k}} M_{l}=N_{i j k}^{l} \equiv \sum_{m \in \mathcal{I}} N_{i j}^{m} N_{m k}^{l} \equiv \operatorname{dim}_{\mathbb{C}} \operatorname{Hom}_{\mathcal{C}}\left(U_{i} \otimes U_{j} \otimes U_{k}, U_{l}\right) .
$$

Note that these non-negative integers are the dimensions of four-point conformal blocks. The same dimensions appear in the fusion rules of permutation orbifold theories [BHS]. This does not come as a surprise: from the point of view of the permutation orbifold, the algebra in Proposition 13 is Morita equivalent to the tensor unit, and computing the annulus coefficients with the latter algebra, one gets directly the fusion rules of the orbifold theory.

At the same time, our results establish that the modular invariant for the tensor product of an arbitrary finite number $N$ of identical chiral conformal field theories obtained from any permutation $g \in \mathfrak{S}_{N}$ is physical. (Such modular invariants have been considered in string theory, see e.g. [FKS].) This follows from the fact that every permutation in $\mathfrak{S}_{N}$ is the product of transpositions, and that the torus partition function obtained from a tensor product of special symmetric Frobenius algebras is given by the matrix product, see Proposition 5.3 of [FRS1]. We summarise these findings in

Corollary 22. Every permutation modular invariant for a rational conformal field theory with chiral symmetry $\mathcal{V} \otimes_{\mathbb{C}} \ldots \otimes_{\mathbb{C}} \mathcal{V}$ is physical, i.e. there exists a consistent full local conformal field theory that has this modular invariant as its torus partition function (and it can be obtained via the construction of [FRS1]).

For instance, it is easy to see that $\mathcal{C}$ has the structure of a module category over $\mathcal{C} \otimes \mathcal{C} \nabla \cdots \otimes \mathcal{C}(m$ factors) for which

$$
\underline{\text { End }}(\mathbf{1}) \cong \bigoplus_{i_{1}, i_{2}, \ldots, i_{m} \in \mathcal{I}} N_{i_{1}, i_{2}, \ldots, i_{m}} U_{i_{1}} \times U_{i_{2}} \times \cdots \times U_{i_{m}}
$$

as an object of $\mathcal{C}$.

Let us also point out that already remark 21 hints at a relation with permutation orbifolds. Indeed, the permutation group $\mathfrak{S}_{N}$ acts naturally on the $N$-fold tensor product $\mathcal{V} \otimes_{\mathbb{C}} \ldots \otimes_{\mathbb{C}} \mathcal{V}$ of vertex algebras. By general arguments one expects the invariant subalgebra, the so-called permutation orbifold chiral algebra, to be a rational vertex algebra as well. Its representation category, the modular tensor category $\mathcal{C}^{\text {orb }}$ is of considerable interest, e.g. because its data enter all known approaches to the congruence subgroup conjecture (see [Ba1, Ba2]). This category cannot be obtained from $\mathcal{C}^{\otimes N}$ alone; rather, an $\mathfrak{S}_{N}$-equivariant modular category with $\mathcal{C}^{\boxplus N}$ as its neutral component must be given. Our results provide the first steps in this direction:

Remark 23. Recall that, for $\mathcal{C}$ a braided monoidal category and any $n, \hat{n} \in \mathbb{Z}, \mathcal{C}$ together with $\left(\bigotimes, \psi^{(n)}, r\right)$ as in Theorem 2 and with $\left(\widehat{\otimes}, \widehat{\psi}^{(\hat{n})}, l\right)$ as in Corollary 3 is both a left and a right module category over $\mathcal{C} \times \mathcal{C}$. Actually, together with suitable natural mixed associativity isomorphisms

$$
\widetilde{\psi}_{X, M, Y}^{(n, \hat{n})}: X \widehat{\otimes}(M \otimes Y) \rightarrow(X \widehat{\otimes} M) \otimes Y
$$

it is even a bimodule category over $\mathcal{C} \times \mathcal{C}$. The mixed associator is given by

$$
\widetilde{\psi}_{X, M, Y}^{(n, \hat{n})}:=\widehat{\gamma}_{X, M Y}^{\hat{n}} \circ\left[i d_{X} \widehat{\otimes} \gamma_{M, Y}^{n}\right] \circ\left[\widehat{\gamma}_{X, M}^{-\hat{n}} \otimes i d_{Y}\right] \circ \gamma_{X M, Y}^{-n}
$$

with

$$
\gamma_{M, U \times V}:=D_{M, U}^{-1} \otimes i d_{V} \quad \text { and } \quad \widehat{\gamma}_{U \times V, M}:=i d_{U} \otimes D_{V, M} .
$$

If $\mathcal{C}$ has in addition a twist, then for all $n, \hat{n} \in \mathbb{Z}$ the so obtained structures of bimodule category on $\mathcal{C}$ are equivalent. This will be discussed in more detail elsewhere. 


\section{Acknowledgements}

We thank Alexei Davydov for helpful discussions. JF is partially supported by VR under project no. 621-2006-3343. IR is partially supported by the EPSRC First Grant EP/E005047/1 and the Marie Curie network 'Superstring Theory' (MRTN-CT-2004-512194). TB and CS are partially supported by the Collaborative Research Centre 676 "Particles, Strings and the Early Universe the Structure of Matter and Space-Time".

\section{References}

[Ba1] P. Bantay, The kernel of the modular representation and the Galois action in RCFT, Commun. Math. Phys. 233 (2003), 423-438 [math.QA/0102149]

[Ba2] P. Bantay, Permutation orbifolds and their applications, Fields Inst. Commun. 39 (2003), 13-23 [hep-th/0104203]

[BE] J. Böckenhauer and D.E. Evans, Modular invariants, graphs, and $\alpha$-induction for nets of subfactors, Commun. Math. Phys. 197 (1998), 361-386 [hep-th/9801171]

[BHS] L.A. Borisov, M.B. Halpern, and C. Schweigert, Systematic approach to cyclic orbifolds, Int. J. Mod. Phys. A 13 (1998), 125-168 [hep-th/9701061]

[BK] B. Bakalov and A.A. Kirillov, Lectures on Tensor Categories and Modular Functors (American Mathematical Society, Providence 2001)

[Br] A. Bruguières, Catégories prémodulaires, modularisations et invariants des variétés de dimension 3, Math. Annal. 316 (2000), 215-236

[ENO] P.I. Etingof, D. Nikshych, and V. Ostrik, On fusion categories, Ann. Math. 162 (2005), 581-642 [math.QA/0203060]

[FB] E. Frenkel and D. Ben-Zvi, Vertex Algebras and Algebraic Curves, second edition (American Mathematical Society, Providence 2004)

[FjFRS1] J. Fjelstad, J. Fuchs, I. Runkel, and C. Schweigert, TFT construction of RCFT correlators V: Proof of modular invariance and factorisation, Theory and Appl. Cat. 16 (2006), 342-433 [hep-th/0503194]

[FjFRS2] J. Fjelstad, J. Fuchs, I. Runkel, and C. Schweigert, Uniqueness of open/closed rational CFT with given algebra of open states, Adv. Theor. Math. Phys. 12 (2008), 1281-1373 [hep-th/0612306]

[FFRS] J. Fröhlich, J. Fuchs, I. Runkel and C. Schweigert, Correspondences of ribbon categories, Adv. in Math. 199 (2006), 192-329 [math.CT/0309465]

[FKS] J. Fuchs, A. Klemm, and M.G. Schmidt, Orbifolds by cyclic permutations in Gepner type superstrings and in the corresponding Calabi-Yau manifolds, Ann. Phys. 214 (1992), $221-257$ 
[FRS1] J. Fuchs, I. Runkel and C. Schweigert, TFT construction of RCFT correlators I: Partition functions, Nucl. Phys. B 646 (2002), 353-497 [hep-th/0204148]

[FRS2] J. Fuchs, I. Runkel and C. Schweigert, Ribbon categories and (unoriented) CFT: Frobenius algebras, automorphisms, reversions, Contemp. Math. 431 (2007), 203-224 [math.CT/0511590]

[FRS3] J. Fuchs, I. Runkel, and C. Schweigert, The fusion algebra of bimodule categories, Applied Categorical Structures 16 (2008), 123-140 [math.CT/0701223]

[Fr] E. Frenkel, Vertex algebras and algebraic curves, Séminaire Bourbaki No. 875 (19992000), 1-41 [math.QA/0007054]

[Ga] T. Gannon, Modular data: the algebraic combinatorics of conformal field theory, J. Algebraic Combin. 22 (2005), 211-250 [math.QA/0103044]

[Hu] Y.-Z. Huang, Vertex operator algebras, the Verlinde conjecture and modular tensor categories, Proc. Natl. Acad. Sci. USA 102 (2005), 5352-5356 [math.QA/0412261]

[JS] A. Joyal and R. Street, Braided tensor categories, Adv. in Math. 102 (1993), 20-78

[Ka] C. Kassel, Quantum Groups (Springer Verlag, New York 1995)

[Ki] A.A. Kirillov, On G-equivariant modular categories, preprint math.QA/0401119

[KR1] L. Kong and I. Runkel, Morita classes of algebras in modular tensor categories, Adv. in Math. 219 (2008), 1548-1576 [0708.1897]

[KR2] L. Kong and I. Runkel, Cardy algebras and sewing constraints, I, preprint 0807.3356

[LR] R. Longo and K.-H. Rehren, Nets of subfactors, Rev. Math. Phys. 7 (1995), 567-598 [hep-th/9411077]

[Mü1] M. Müger, From subfactors to categories and topology I. Frobenius algebras in and Morita equivalence of tensor categories, J. Pure Appl. Alg. 180 (2003), 81-157 [math.CT/0111204]

[Mü2] M. Müger, Conformal orbifold theories and braided crossed G-categories, Commun. Math. Phys. 260 (2005), 727-762 [math.QA/0403322] [ibid. 260 (2005) 763, Erratum]

[Os] V. Ostrik, Module categories, weak Hopf algebras and modular invariants, Transform. Groups 8 (2003), 177-206 [math.QA/0111139]

[SFR] C. Schweigert, J. Fuchs, and I. Runkel, Categorification and correlation functions in conformal field theory, in: Proceedings of the ICM 2006 (M. Sanz-Solé, J. Soria, J.L. Varona, and J. Verdera, eds.) (European Mathematical Society, Zürich 2006), p. 443458 [math.CT/0602079]

[SS] N. Sousa and A.N. Schellekens, Orientation matters for NIMreps, Nucl. Phys. B 653 (2003), 339-368 [hep-th/0210014] 
[Tu] V.G. Turaev, Homotopy field theory in dimension 3 and crossed group-categories, preprint math.GT/0005291

[VZ] F. Van Oystaeyen and Y.H. Zhang, The Brauer group of a braided monoidal category, J. Algebra 202 (1998), 96-128

[Xu] F. Xu, New braided endomorphisms from conformal inclusions, Commun. Math. Phys. 192 (1998), 349-403 